\title{
Injective Envelopes and Local Multiplier Algebras of Some Spatial Continuous Trace $\mathrm{C}^{*}$-algebras*
}

\author{
Martín Argerami, Douglas Farenick, and Pedro Massey \\ Department of Mathematics and Statistics, University of Regina \\ Regina, Saskatchewan S4S 0A2, Canada \\ Departamento de Matemática, Facultad de Ciencias Exactas \\ Universidad Nacional de La Plata, Argentina
}

May 28, 2013

\begin{abstract}
A precise description of the injective envelope of a spatial continuous trace $\mathrm{C}^{*}$-algebra $A$ over a Stonean space $\Delta$ is given. The description is based on the notion of a weakly continuous Hilbert bundle, which we show herein to be a Kaplansky-Hilbert module over the abelian $\mathrm{AW}^{*}$-algebra $C(\Delta)$. We then use the description of the injective envelope of $A$ to study the first- and second-order local multiplier algebras of $A$. In particular, we show that the second-order local multiplier algebra of $A$ is precisely the injective envelope of $A$.
\end{abstract}

\section{Introduction}

A commonly used technique in the theory of operators algebras is to study a given $\mathrm{C}^{*}$-algebra $A$ by one or more of its enveloping algebras. Well known examples of such enveloping algebras are the enveloping von Neumann algebra $A^{* *}$ and the multiplier algebra $M(A)$. In this paper we consider two others: the local multiplier algebra $M_{\text {loc }}(A)$ and the injective envelope $I(A)$, both

*2000 Mathematics Subject Classification: Primary 46L05; Secondary 46L07. Keywords and Phrases: local multiplier algebra, injective envelope, continuous trace $\mathrm{C}^{*}$ algebra, continuous Hilbert bundle. This work is supported in part by the NSERC Discovery Grant Program (Canada) and CONICET (Argentina). 
of which have received considerable study and application in recent years (see, for example, $[1,6,7,9,11,19,21,22]$ ).

The $\mathrm{C}^{*}$-algebras $M_{\mathrm{loc}}(A)$ and $I(A)$ are difficult to determine precisely, even for fairly rudimentary types of $\mathrm{C}^{*}$-algebras $A$. For instance, if we denote by $C_{0}(T)$ an abelian $\mathrm{C}^{*}$-algebra and by $K(H)$ the ideal of compact operators over $H$, their local multiplier algebra and injective envelope have been readily computed; but the injective envelope of $C_{0}(T) \otimes K(H)$ is much more difficult to describe: see [15] for an abstract description and [3, 4] for a somewhat more concrete one.

Our first goal in the present paper is to make a further contribution to the issue of the determination of $I(A)$ and $M_{\text {loc }}(A)$ from $A$ by considering continuous trace $\mathrm{C}^{*}$-algebras studied by Fell [10] that arise from continuous Hilbert bundles. The class of such algebras contains in particular all $\mathrm{C}^{*}$ algebras of the form $C_{0}(T) \otimes K(H)$, which were studied in [4]. Because the centres of $I(A)$ and $M_{\mathrm{loc}}(A)$ are $\mathrm{AW}^{*}$-algebras, and thus have Stonean maximal ideal spaces, we restrict ourselves in this paper to locally compact Hausdorff spaces $T$ that are Stonean. In so doing, we establish an important first step toward a complete analysis, in the case of non-Stonean $T$, of the $\mathrm{C}^{*}$-algebras $I(A), M_{\mathrm{loc}}(A)$, and $M_{\mathrm{loc}}\left(M_{\mathrm{loc}}(A)\right)$ for spatial continuous trace $\mathrm{C}^{*}$-algebras $A$ with spectrum $T$. As the passage from general $T$ to Stonean $T$ involves a number of technicalities, the application of the main results herein to the case of arbitrary locally compact Hausdorff spaces $T$ will be deferred to a subsequent article.

Our second goal is to study and use the notion of a weakly continuous Hilbert bundle $\Omega_{\mathrm{wk}}$ relative to a continuous Hilbert bundle $\Omega$ over a locally compact Hausdorff space $T$. Particular cases of this notion have been previously considered in $[15,23]$. It is natural to consider $\Omega$ as a $\mathrm{C}^{*}$-module over the abelian $\mathrm{C}^{*}$-algebra $C_{0}(T)$; if, moreover, $T$ is a Stonean space $\Delta$, we then show $\Omega_{\mathrm{wk}}$ carries the structure of a faithful $\mathrm{AW}^{*}$-module over $C(\Delta)$. In this latter situation, such $\mathrm{C}^{*}$-modules are called Kaplansky-Hilbert modules. We study the $\mathrm{C}^{*}$-modules $\Omega$ and $\Omega_{\mathrm{wk}}$, as well as certain $\mathrm{C}^{*}$-algebras of endomorphisms of these modules, using the beautiful machinery Kaplansky developed in his seminal work from the early 1950s [16]. In particular, we prove that the $\mathrm{C}^{*}$-algebra $B\left(\Omega_{\mathrm{wk}}\right)$ of bounded adjointable endomorphisms of $\Omega_{\mathrm{wk}}$ is the injective envelope and second-order local multiplier algebra of the $\mathrm{C}^{*}$-algebra $K(\Omega)$ of "compact" endomorphisms of $\Omega$.

Assuming that $T=\Delta$, a Stonean space, and in postponing the precise definitions until the following section, we summarise in this paragraph the main results of the paper. In Section 2, we show that $\Omega_{\mathrm{wk}}$ is a KaplanskyHilbert module that contains $\Omega$ as a $\mathrm{C}^{*}$-submodule such that $\Omega^{\perp}=\{0\}$. In 
Section 3, we prove that $B\left(\Omega_{\mathrm{wk}}\right)$ is the injective envelope of both $K(\Omega)$ and the Fell continuous trace $C^{*}$-algebra $A$ induced by the bundle $\Omega$. Section 4 deals with local multipliers, and we show that $B\left(\Omega_{\mathrm{wk}}\right)$ is the second-order local multiplier algebra of both $K(\Omega)$ and Fell algebra $A$. We also prove that the equality $M_{\mathrm{loc}}\left(M_{\mathrm{loc}}(A)\right)=I(A)$ holds for certain type I non-separable $\mathrm{C}^{*}$-algebras, generalising a result of Somerset [21]. Finally, in Section 5 we find that a direct-sum decomposition of $\Omega_{\mathrm{wk}}$ leads to a corresponding decomposition of (the generally non- $\mathrm{AW}^{*}$ ) algebra $M_{\mathrm{loc}}(A)$ but not to a decomposition of $A$.

\section{Preliminaries}

If $T$ is a locally compact Hausdorff space and $\left\{H_{t}\right\}_{t \in T}$ is family of Hilbert spaces, a vector field on $T$ with fibres $H_{t}$ is a function $\nu: T \rightarrow \bigsqcup_{t} H_{t}$ in which $\nu(t) \in H_{t}$, for every $t \in T$. Such a vector field $\nu$ is said to be bounded if the function $t \mapsto\|\nu(t)\|$ is bounded. From this point on, the notation $T \rightarrow \bigsqcup_{t} H_{t}$ will be taken to also imply that, for all $t$, the point $t$ is mapped into the corresponding fibre $H_{t}$.

Definition 1.1. A continuous Hilbert bundle [8] is a triple $\left(T,\left\{H_{t}\right\}_{t \in T}, \Omega\right)$, where $\Omega$ is a set of vector fields on $T$ with fibres $H_{t}$ such that:

(I) $\Omega$ is a $C(T)$-module with the action $(f \cdot \omega)(t)=f(t) \omega(t)$;

(II) for each $t_{0} \in T,\left\{\omega\left(t_{0}\right): \omega \in \Omega\right\}=H_{t_{0}}$;

(III) the map $t \mapsto\|\omega(t)\|$ is continuous, for all $\omega \in \Omega$;

(IV) $\Omega$ is closed under local uniform approximation-that is, if $\xi: T \rightarrow$ $\bigsqcup_{t} H_{t}$ is any vector field such that for every $t_{0} \in T$ and $\varepsilon>0$ there is an open set $U \subset T$ containing $t_{0}$ and $a \omega \in \Omega$ with $\|\omega(t)-\xi(t)\|<\varepsilon$ for all $t \in U$, then necessarily $\xi \in \Omega$.

Dixmier and Douady [8] show that (I), (II), and (IV) can be replaced by other axioms, such as those given by Fell [10], without altering the structure that arises. For example, in the presence of the other axioms, (II) is equivalent to " $\left\{\omega\left(t_{0}\right): \omega \in \Omega\right\}$ is dense in $H_{t_{0}}$, for each $t_{0} \in T$ "; in the presence of (IV), axiom (I) can be replaced by " $\Omega$ is a complex vector space".

We turn next to the notion of a weakly continuous Hilbert bundle. If $\left(T,\left\{H_{t}\right\}_{t \in T}, \Omega\right)$ is a continuous Hilbert bundle then, by the polarisation identity, the function $t \mapsto\left\langle\omega_{1}(t), \omega_{2}(t)\right\rangle$ is continuous for all $\omega_{1}, \omega_{2} \in \Omega$. In defining $\left\langle\omega_{1}, \omega_{2}\right\rangle$ to be the map $T \rightarrow \mathbb{C}$ given by $t \mapsto\left\langle\omega_{1}(t), \omega_{2}(t)\right\rangle$, one 
obtains a $C(T)$-valued inner product on $\Omega$ which gives $\Omega$ the structure of an inner product module over $C(T)$.

Definition 1.2. A vector field $\nu: T \rightarrow \bigsqcup_{t} H_{t}$ is said to be weakly continuous with respect to the continuous Hilbert bundle $\left(T,\left\{H_{t}\right\}_{t \in T}, \Omega\right)$ if the function

$$
t \longmapsto\langle\nu(t), \omega(t)\rangle
$$

is continuous for all $\omega \in \Omega$. The set of all bounded weakly continuous vector fields with respect to a given $\Omega$ will be denoted by $\Omega_{\mathrm{wk}}$, that is

$$
\Omega_{\mathrm{wk}}=\left\{\nu: T \rightarrow \bigsqcup_{t} H_{t}: \sup _{t}\|\nu(t)\|<\infty \text { and } \nu \text { is weakly continuous }\right\} .
$$

We will call the quadruple $\left(T,\left\{H_{t}\right\}_{t \in T}, \Omega, \Omega_{\mathrm{wk}}\right)$ a weakly continuous Hilbert bundle over $T$.

We remark that when $T$ is compact, $\Omega_{\mathrm{wk}}$ is a $C(T)$-module under the pointwise module action, and also $\Omega \subset \Omega_{\mathrm{wk}}$ (because then every continuous field on $T$ is bounded). However, the function $t \mapsto\left\langle\nu_{1}(t), \nu_{2}(t)\right\rangle$ is generally not continuous for arbitrary $\nu_{1}, \nu_{2} \in \Omega_{\mathrm{wk}}$. Thus, although $\Omega_{\mathrm{wk}}$ is, algebraically, a module over $C_{b}(T)$, it is not in general an inner product module over $C_{b}(T)$. Nevertheless, if $T$ has the right topology-namely that of a Stonean space - then we show (Theorem 2.6) that it is possible to endow a weakly continuous Hilbert bundle with the structure of a $\mathrm{C}^{*}$-module over the $\mathrm{C}^{*}$-algebra of continuous complex-valued functions on $T$.

The continuous trace $\mathrm{C}^{*}$-algebras we consider herein were first studied by Fell [10]. We now recall their definition.

Assume that $\left\{A_{t}\right\}_{t \in T}$ is a family of $\mathrm{C}^{*}$-algebras indexed by the locally compact Hausdorff topological space $T$. An operator field is a map $a: T \rightarrow$ $\bigsqcup_{t} A_{t}$ such that $a(t) \in A_{t}$, for each $t \in T$.

Definition 1.3. Let $\left(T,\left\{H_{t}\right\}_{t \in T}, \Omega\right)$ be a continuous Hilbert bundle. An operator field $a: T \rightarrow \bigsqcup_{t \in T} K\left(H_{t}\right)$ is:

$i$. almost finite-dimensional (with respect to $\Omega$ ) if for each $t_{0} \in T$ and $\varepsilon>0$ there exist an open set $U \subset T$ containing $t_{0}$ and $\omega_{1}, \ldots, \omega_{n} \in \Omega$ such that

(a) $\omega_{1}(t), \ldots, \omega_{n}(t)$ are linearly independent for every $t \in U$, and

(b) $\left\|p_{t} a(t) p_{t}-a(t)\right\|<\varepsilon$ for all $t \in U$, where $p_{t} \in B\left(H_{t}\right)$ is the projection with range $\operatorname{Span}\left\{\omega_{j}(t): 1 \leq j \leq n\right\}$; 
ii. weakly continuous (with respect to $\Omega$ ) if the complex-valued function

$$
t \longmapsto\left\langle a(t) \omega_{1}(t), \omega_{2}(t)\right\rangle
$$

is continuous for every $\omega_{1}, \omega_{2} \in \Omega$.

Definition 1.4. ([10]) Let $\left(T,\left\{H_{t}\right\}_{t \in T}, \Omega\right)$ be a continuous Hilbert bundle. The Fell algebra of the Hilbert bundle $\left(T,\left\{H_{t}\right\}_{t \in T}, \Omega\right)$, denoted by $A=A\left(T,\left\{H_{t}\right\}_{t \in T}, \Omega\right)$, is the set of all weakly continuous, almost finitedimensional operator fields $a: T \rightarrow \bigsqcup_{t \in T} K\left(H_{t}\right)$ for which $t \mapsto\|a(t)\|$ is continuous and vanishes at infinity, endowed with pointwise operations and norm

$$
\|a\|=\max _{t \in T}\|a(t)\|, \quad a \in A .
$$

We shall make repeated use of the following fact about the Fell algebras of Hilbert bundles: if $A=A\left(T,\left\{H_{t}\right\}_{t \in T}, \Omega\right)$, for some continuous Hilbert bundle $\left(T,\left\{H_{t}\right\}_{t \in T}, \Omega\right)$, then $A$ is a continuous trace $\mathrm{C}^{*}$-algebra with spectrum $\hat{A} \simeq T[10$, Theorems 4.4, 4.5].

\section{An $\mathrm{AW}^{*}$-module Structure for $\Omega_{\mathrm{wk}}$}

Assume henceforth that $T=\Delta$ is a Stonean space; that is, $\Delta$ is Hausdorff, compact, and extremely disconnected. The abelian $\mathrm{C}^{*}$-algebra $C(\Delta)$ is an $\mathrm{AW}^{*}$-algebra and so one may ask whether the $\mathrm{C}^{*}$-modules $\Omega$ and $\Omega_{\mathrm{wk}}$ are $\mathrm{AW}^{*}$-modules in the sense of Kaplansky [16]. We shall show that this is indeed true for the module $\Omega_{\mathrm{wk}}$. As a consequence of this last fact we shall get that the $\mathrm{C}^{*}$-algebra $B\left(\Omega_{\mathrm{wk}}\right)$ of bounded adjointable endomorphisms of $\Omega_{\mathrm{wk}}$ is an $\mathrm{AW}^{*}$-algebra of type I.

The following lemmas are needed to describe the $C(\Delta)$-Hilbert module structure of $\Omega_{\mathrm{wk}}$.

Lemma 2.1. Let $f: \Delta \rightarrow \mathbb{R}$ be a lower semicontinuous function such that there exist $g \in C(\Delta)$ and a meagre set $M \subset \Delta$ with $f(s)=g(s)$ for all $s \in \Delta \backslash M$. Then

$$
\sup _{s \in \Delta} g(s)=\sup _{s \in \Delta \backslash M} f(s)=\sup _{s \in \Delta} f(s) .
$$

Proof. Let $\rho=\sup _{s \in \Delta \backslash M} f(s)=\sup _{s \in \Delta \backslash M} g(s) \leq \sup _{s \in \Delta} g(s)$; then $f(s) \leq \rho$ for all $s \in \Delta \backslash M$. Because $\Delta$ is a Baire space, $\overline{\Delta \backslash M}=\Delta$; thus, by the lower semi-continuity, $f(s) \leq \rho$ for every $s \in \Delta$. The same argument yields that $g(s) \leq \rho$ for all $s \in \Delta$. 
Lemma 2.2. Assume that $\left(\Delta,\left\{H_{s}\right\}_{s \in \Delta}, \Omega\right)$ is a continuous Hilbert bundle and $\nu \in \Omega_{\mathrm{wk}}$. Then

i. the function $s \mapsto\|\nu(s)\|^{2}$ is lower semicontinuous;

ii. there is a meagre subset $M \subset \Delta$ and a continuous function $h: \Delta \rightarrow \mathbb{R}_{+}$ such that

(a) $h(s)=\|\nu(s)\|^{2}$ for all $s \in \Delta \backslash M$, and

(b) $\|h\|=\sup _{s \in \Delta \backslash M}\|\nu(s)\|^{2}=\sup _{s \in \Delta}\|\nu(s)\|^{2}$.

Proof. Let $r \in \mathbb{R}$ be fixed and consider $U_{r}=\left\{s \in \Delta: r<\|\nu(s)\|^{2}\right\}$. We aim to show that $U_{r}$ is open. Choose $s_{0} \in U_{r}$. Thus, $r<\left\|\nu\left(s_{0}\right)\right\|^{2}$. By Parseval's formula, there are orthonormal vectors $\xi_{1}, \ldots, \xi_{n} \in H_{s_{0}}$ such that $r<$ $\sum_{j=1}^{n}\left|\left\langle\nu\left(s_{0}\right), \xi_{j}\right\rangle\right|^{2} \leq\left\|\nu\left(s_{0}\right)\right\|^{2}$. Choose any $\mu_{1}, \ldots, \mu_{n} \in \Omega$ such that $\mu_{j}\left(s_{0}\right)=$ $\xi_{j}$, for each $j$. Because $\xi_{1}, \ldots, \xi_{n}$ are orthogonal, $\mu_{1}(s), \ldots, \mu_{n}(s)$ are linearly independent in an open neighbourhood of $s_{0}$. Hence, by [10, Lemma 4.2], there is an open set $V$ containing $s_{0}$ and vector fields $\omega_{1}, \ldots, \omega_{n} \in \Omega$ such that $\omega_{1}(s), \ldots, \omega_{n}(s)$ are orthonormal for all $s \in V$, and $\omega_{j}\left(s_{0}\right)=\xi_{j}$ for each $j$. The function

$$
g(s)=\sum_{j=1}^{n}\left|\left\langle\nu(s), \omega_{j}(s)\right\rangle\right|^{2}
$$

on $\Delta$ is continuous and satisfies $g(s) \leq\|\nu(s)\|^{2}$, for every $s \in V$, and $r<g\left(s_{0}\right)$. Therefore, by the continuity of $g$, there is an open set $W \subset V$ containing $s_{0}$ such that $r<g(s) \leq\|\nu(s)\|^{2}$ for all $s \in W$. This proves that $U_{r}$ contains an open set around each of its points. That is, $U_{r}$ is open.

Because every bounded nonnegative lower semicontinuous function on a Stonean space $\Delta$ agrees with a nonnegative continuous function off a meagre set $M$ [24, Proposition III.1.7], the function $h \in C(\Delta)$ as in (ii) exists and satisfies $h(s)=\|\nu(s)\|^{2}$ for $s \in \Delta \backslash M$.

The last statement follows from Lemma 2.1.

Let $\left(\Delta,\left\{H_{t}\right\}_{t \in \Delta}, \Omega, \Omega_{\mathrm{wk}}\right)$ be a weakly continuous Hilbert bundle over $\Delta$. Given $\nu \in \Omega_{\mathrm{wk}}$, the function $h$ that arises in Lemma 2.2 will be denoted by $\langle\nu, \nu\rangle$. There is no ambiguity in so doing because if $h_{1}, h_{2} \in C(\Delta)$ and if $h_{1}(s)=h_{2}(s)$ for all $s \notin\left(M_{1} \cup M_{2}\right)$ for some meagre subsets $M_{1}$ and $M_{2}$, then $h_{1}$ and $h_{2}$ agree on $\Delta$. (If not, then by continuity, $h_{1}$ and $h_{2}$ would 
differ on an open set $U$; but $\emptyset \neq U \subset M_{1} \cup M_{2}$ is in contradiction to the fact that no meagre set in a Baire space can contain a nonempty open set.)

Now use the polarisation identity to define $\left\langle\nu_{1}, \nu_{2}\right\rangle \in C(\Delta)$ for any pair $\nu_{1}, \nu_{2} \in \Omega_{\mathrm{wk}}$. This gives $\Omega_{\mathrm{wk}}$ the structure of pre-inner product module over $C(\Delta)$ whereby for each $\nu_{1}, \nu_{2} \in \Omega_{\mathrm{wk}}$ there is a meagre subset $M_{\nu_{1}, \nu_{2}} \subset \Delta$ such that the continuous function $\left\langle\nu_{1}, \nu_{2}\right\rangle$ satisfies

$$
\left\langle\nu_{1}, \nu_{2}\right\rangle(s)=\left\langle\nu_{1}(s), \nu_{2}(s)\right\rangle, \quad \forall s \in \Delta \backslash M_{\nu_{1}, \nu_{2}} .
$$

In particular, if $\nu \in \Omega_{\mathrm{wk}}$ and $\omega \in \Omega$, then

$$
\langle\nu, \omega\rangle(s)=\langle\nu(s), \omega(s)\rangle, \quad \forall s \in \Delta .
$$

In fact, $\Omega_{\mathrm{wk}}$ is an inner product module over $C(\Delta)$, for if $\nu \in \Omega_{\mathrm{wk}}$ satisfies $\langle\nu, \nu\rangle=0$, then Lemma 2.2 yields $\|\nu(s)\|^{2}=0$ for all $s \in \Delta$. Therefore,

$$
\|\nu\|=\|\langle\nu, \nu\rangle\|^{1 / 2}, \quad \nu \in \Omega_{\mathrm{wk}},
$$

defines a norm on $\Omega_{\mathrm{wk}}$, where

$$
\|\nu\|^{2}=\sup _{s \in \Delta}\langle\nu(s), \nu(s)\rangle=\|\langle\nu, \nu\rangle\| .
$$

Recall that given a $\mathrm{C}^{*}$-algebra $B$, a Hilbert $C^{*}$-module over $B$ is a left $B$-module $E$ together with a $B$-valued definite sequilinear map $\langle$,$\rangle such$ that $E$ is complete with the norm $\|\nu\|=\|\langle\nu, \nu\rangle\|^{1 / 2}$ (we refer to [17] for a detailed account on Hilbert modules).

Note that if $\nu \in \Omega_{\mathrm{wk}}$, then $|\nu|(s):=\langle\nu, \nu\rangle^{1 / 2}(s) \geq\|\nu(s)\|$ for $s \in \Delta$ and there exists a meagre set $M \subset \Delta$ with $|\nu|(s)=\|\nu(s)\|$ if $s \in(\Delta \backslash M)$ (Lemma 2.2). These facts will be used repeatedly from now on.

Proposition 2.3. $\Omega_{\mathrm{wk}}$ is a $C^{*}$-module over $C(\Delta)$ and $\Omega$ is a $C^{*}$-submodule of $\Omega_{\mathrm{wk}}$.

Proof. The only Hilbert $\mathrm{C}^{*}$-module axiom that is not obviously satisfied by $\Omega_{\mathrm{wk}}$ is the axiom of completeness. Let $\left\{\nu_{i}\right\}_{i \in \mathbb{N}}$ be a Cauchy sequence in $\Omega_{\mathrm{wk}}$. By the equality (1), $\left\{\nu_{i}(s)\right\}_{i \in \mathbb{N}}$ is a Cauchy sequence in $H_{s}$ for every $s \in \Delta$. Let $\nu(s) \in H_{s}$ denote the limit of this sequence so that $\nu: \Delta \rightarrow \bigsqcup_{s \in \Delta} H_{s}$ is a vector field.

Choose $\omega \in \Omega$ and consider the function $g_{i, \omega} \in C(\Delta)$ given by $g_{i, \omega}(s)=$ $\left\langle\omega(s), \nu_{i}(s)\right\rangle$. Let $\varepsilon>0$. Then there is $N_{\varepsilon} \in \mathbb{N}$ such that $\left\|\nu_{i}-\nu_{j}\right\|<\varepsilon$, for all $i, j \geq N_{\varepsilon}$. Therefore, the Cauchy-Schwarz inequality yields

$$
\sup _{s \in \Delta}\left|g_{i, \omega}(s)-g_{j, \omega}(s)\right|<\varepsilon\|\omega\|, \quad \forall i, j \geq N_{\varepsilon} .
$$


Thus, the sequence $\left\{g_{i, \omega}\right\}_{i}$ is Cauchy in $C(\Delta)$; let $g_{\omega} \in C(\Delta)$ denote its limit. Observe that $g_{\omega}(s)=\lim _{i}\left\langle\nu_{i}(s), \omega(s)\right\rangle=\langle\nu(s), \omega(s)\rangle$, for all $s \in \Delta$. As the choice of $\omega \in \Omega$ is arbitrary, this shows that $\nu$ is weakly continuous. The Cauchy sequence $\left\{\nu_{i}\right\}_{i \in \mathbb{N}}$ is necessarily uniformly bounded by, say, $\rho>0$, and then $\|\nu(s)\| \leq \rho$ for every $s \in \Delta$. That is, the function $s \rightarrow\|\nu(s)\|$ is bounded and so $\nu \in \Omega_{\mathrm{wk}}$. Finally, if $i, j \geq N_{\varepsilon}$, then for any $s \in \Delta$ we have $\left\|\nu(s)-\nu_{i}(s)\right\| \leq\left\|\nu(s)-\nu_{j}(s)\right\|+\left\|\nu_{j}(s)-\nu_{i}(s)\right\| \leq\left\|\nu(s)-\nu_{j}(s)\right\|+\varepsilon$, and so letting $j \rightarrow \infty$ yields $\left\|\nu(s)-\nu_{i}(s)\right\| \leq \varepsilon$ for every $s \in \Delta$. That is, $\left\|\nu-\nu_{i}\right\| \rightarrow 0$, which proves that $\Omega_{\mathrm{wk}}$ is complete.

For the case of $\Omega$, let $\left\{\omega_{n}\right\}_{n \in \mathbb{N}}$ be a Cauchy sequence in $\Omega$. For each $s \in \Delta,\left\{\omega_{n}(s)\right\}_{n \in \mathbb{N}}$ is a Cauchy sequence in $H_{s}$; let $\omega(s)$ denote the limit. Since the limit is uniform, it is in particular locally uniform, and so $\omega \in \Omega$. Hence, $\Omega$ is complete.

Definition 2.4. A Hilbert $C^{*}$-module $E$ over a $C^{*}$-algebra $B$ is called a Kaplansky-Hilbert module if in addition $B$ is an abelian $A W^{*}$-algebra and the following three properties hold [16, p. 842] (Kaplansky's original term for such a module was "faithful $A W^{*}$-module"):

i. if $e_{i} \cdot \nu=0$ for some family $\left\{e_{i}\right\}_{i} \subset B$ of pairwise-orthogonal projections and $\nu \in E$, then also $e \cdot \nu=0$, where $e=\sup _{i} e_{i}$;

ii. if $\left\{e_{i}\right\}_{i} \subset B$ is a family of pairwise-orthogonal projections such that $1=\sup _{i} e_{i}$, and if $\left\{\nu_{i}\right\}_{i} \subset E$ is a bounded family, then there is a $\nu \in E$ such that $e_{i} \cdot \nu=e_{i} \cdot \nu_{i}$ for all $i$;

iii. if $\nu \in E$, then $g \cdot \nu=0$ for all $g \in B$ only if $\nu=0$.

Remark 2.5. The element $\nu \in E$ obtained in the situation described in (ii) will sometimes be denoted as $\sum_{i} e_{i} \nu_{i}$. It should be emphasized that this is not a pointwise sum.

Theorem 2.6. $\Omega_{\mathrm{wk}}$ is a Kaplansky-Hilbert module over $C(\Delta)$.

Proof. For property (i), assume that $\nu \in \Omega_{\mathrm{wk}}$ and $\left\{e_{i}\right\}_{i} \subset C(\Delta)$ is a family of pairwise-orthogonal projections with supremum $e \in C(\Delta)$ for which $e_{i} \cdot \nu=0$ for all $i$. Because projections in $C(\Delta)$ are the characteristic functions of clopen sets, there are pairwise-disjoint clopen sets $U_{i} \subset \Delta$ such that $e_{i}=\chi_{U_{i}}$. Thus, for each $i$, using Lemma 2.2,

$$
\begin{aligned}
0 & =\left\|e_{i} \cdot \nu\right\|^{2}=\max _{s \in \Delta}\left\langle e_{i} \cdot \nu, e_{i} \cdot \nu\right\rangle(s)=\sup _{s \in \Delta}\left\langle e_{i}(s) \nu(s), e_{i}(s) \nu(s)\right\rangle \\
& =\max _{s \in \Delta} e_{i}(s)[\langle\nu, \nu\rangle(s)]=\max _{s \in U_{i}}\langle\nu, \nu\rangle(s),
\end{aligned}
$$


and so $\langle\nu, \nu\rangle(s)=0$ for every $s \in U_{i}$. Let $U=\bigcup_{i} U_{i}$. The set $\bar{U}$ is clopen and $\chi_{\bar{U}}=\sup _{i} e_{i}=e[5, \S 8]$. As $\langle\nu, \nu\rangle$ is a continuous function that vanishes on $U$, it also vanishes on $\bar{U}$. Hence,

$$
\|e \cdot \nu\|^{2}=\max _{s \in \Delta} e(s)[\langle\nu, \nu\rangle(s)]=\max _{s \in \bar{U}}\langle\nu, \nu\rangle(s)=0,
$$

which yields property (i).

For the proof of property (ii), assume that $\left\{e_{i}\right\}_{i} \subset C(\Delta)$ is a family of pairwise-orthogonal projections such that $1=\sup _{i} e_{i}$ and that $\left\{\nu_{i}\right\}_{i} \subset \Omega_{\mathrm{wk}}$ is a family such that $K=\sup \left\|\nu_{i}\right\|<\infty$; we aim to prove that there is a $\nu \in \Omega_{\mathrm{wk}}$ such that $e_{i} \cdot \nu=e_{i} \cdot \nu_{i}$ for all $i$. As before, assume that $e_{i}=\chi_{U_{i}}$ and $U=\bigcup_{i} U_{i}$. Then $1=\sup _{i} e_{i}$ implies that $\bar{U}=\Delta$.

For each $\omega \in \Omega$, consider the unique function $f_{\omega} \in C(\Delta)$ such that $e_{i} f_{\omega}=e_{i}\left\langle\omega, \nu_{i}\right\rangle$ for all $i$ (its existence guaranteed by the fact that $\Delta$ is the Stone-Čech compactification of $U)$. Note that for $s \in U_{i}$ we have that $f_{\omega}(s)=\left\langle\omega(s), \nu_{i}(s)\right\rangle$. Hence, $\left|f_{\omega}(s)\right| \leq K\|\omega(s)\|$ for $s \in U$; the same inequality holds for all $s \in \Delta$ because $\bar{U}=\Delta$ and both sides of the inequality are continuous functions of $s$. Moreover, if $\omega_{1}, \omega_{2} \in \Omega$ and $\alpha \in \mathbb{C}$ then, for $s \in U$ we get that $f_{\alpha \omega_{1}+\omega_{2}}(s)=\alpha f_{\omega_{1}}(s)+f_{\omega_{2}}(s)$ and, therefore, that $f_{\alpha \omega_{1}+\omega_{2}}=\alpha f_{\omega_{1}}+f_{\omega_{2}}$. Thus, for each $s \in \Delta$ the function $\omega(s) \mapsto f_{\omega}(s)$ is a well-defined, bounded linear functional on $H_{s}$. Let $\nu(s) \in H_{s}$ be the representing vector for this functional, yielding a vector field $\nu: \Delta \rightarrow \bigsqcup_{s \in \Delta} H_{s}$. Since $\langle\nu(s), \omega(s)\rangle=\overline{f_{\omega}(s)}$, for every $\omega \in \Omega, \nu$ is weakly continuous. It remains to show that $\nu$ is a bounded vector field. If $s \in U$,

$$
\|\nu(s)\|=\sup _{\omega \in \Omega,\|\omega(s)\|=1}|\langle\omega(s), \nu(s)\rangle|=\sup _{\omega \in \Omega,\|\omega(s)\|=1}\left|f_{\omega}(s)\right| \leq \sup _{i}\left\|\nu_{i}\right\|=K,
$$

which shows that $\|\nu(s)\|$ is uniformly bounded on $U$. Thus, since $U$ is dense, the lower semicontinuous function $s \mapsto\|\nu(s)\|^{2}$ is bounded on $\Delta$. Therefore, $\nu \in \Omega_{\mathrm{wk}}$.

Now we show that $e_{i} \cdot \nu=e_{i} \cdot \nu_{i}$, for all $i$. Fix $i$ and $s \in U_{i}$ and consider $\omega \in \Omega$. Then,

$$
\begin{aligned}
\left\langle\omega(s), e_{i}(s) \nu(s)\right\rangle & =\langle\omega(s), \nu(s)\rangle=f_{\omega}(s) \\
& =e_{i}(s) f_{\omega}(s)=e_{i}(s)\left\langle\omega(s), \nu_{i}(s)\right\rangle \\
& =\left\langle\omega(s), e_{i}(s) \nu_{i}(s)\right\rangle .
\end{aligned}
$$

Since $\left(e_{i} \cdot \nu\right)(s)=0=\left(e_{i} \cdot \nu_{i}\right)$ (s) for $s \in \Delta \backslash U_{i}$ we conclude that $e_{i} \cdot \nu=e_{i} \cdot \nu_{i}$.

For the proof of property (iii), assume that $\nu \in \Omega_{\mathrm{wk}}$ satisfies $g \cdot \nu=0$ for all $g \in C(\Delta)$. Then, in particular, $\langle\nu, \nu\rangle \cdot \nu=0$, so $\langle\nu, \nu\rangle=0$. Hence, from $\|\nu\|=\|\langle\nu, \nu\rangle\|^{1 / 2}=0$ we conclude that $\nu=0$. 


\section{Endomorphisms of $\Omega$ and $\Omega_{\mathrm{wk}}$}

Throughout this section $A$ will denote the Fell $\mathrm{C}^{*}$-algebra of the continuous Hilbert bundle $\left(\Delta,\left\{H_{s}\right\}_{s \in \Delta}, \Omega\right)$, as described in Definition 1.4, with $\Delta$ Stonean. Let $B(\Omega)$ and $B\left(\Omega_{\mathrm{wk}}\right)$ denote, respectively, the $\mathrm{C}^{*}$-algebras of adjointable $C(\Delta)$-endomorphisms of $\Omega$ and $\Omega_{\mathrm{wk}}$. Since, by Theorem 2.6, $\Omega_{\mathrm{wk}}$ is a Kaplansky-Hilbert $\mathrm{AW}^{*}$-module over $C(\Delta), B\left(\Omega_{\mathrm{wk}}\right)$ coincides with the set of all $C(\Delta)$-endomorphisms of $\Omega_{\mathrm{wk}}[16$, Theorem 6$]$ and is a type I $\mathrm{AW}^{*}$-algebra with centre $C(\Delta)[16$, Theorem 7$]$.

In the particular case where $\Omega$ is given by the trivial Hilbert bundle $\left(\Delta,\{H\}_{s \in \Delta}, C(\Delta, H)\right)$ with $H$ is a fixed Hilbert space, Hamana [15] proved that $B\left(\Omega_{\mathrm{wk}}\right) \cong C(\Delta) \bar{\otimes} B(H)$, the monotone complete tensor product of $C(\Delta)$ and $B(H)$.

For each $\nu_{1}, \nu_{2} \in \Omega_{\mathrm{wk}}$, consider the endomorphism $\Theta_{\nu_{1}, \nu_{2}}$ on $\Omega_{\mathrm{wk}}$ defined by

$$
\Theta_{\nu_{1}, \nu_{2}}(\nu)=\left\langle\nu, \nu_{2}\right\rangle \cdot \nu_{1}, \quad \nu \in \Omega_{\mathrm{wk}} .
$$

For a Hilbert bundle $\Omega_{0}$, let

$$
F\left(\Omega_{0}\right)=\left\{\sum_{j=1}^{n} \Theta_{\omega_{j}, \omega_{j}^{\prime}}: n \in \mathbb{N}, \omega_{j}, \omega_{j}^{\prime} \in \Omega\right\} .
$$

We will consider both $F(\Omega)$ and $F\left(\Omega_{\mathrm{wk}}\right)$.

If $\omega_{1}, \omega_{2} \in \Omega$, then $\Theta_{\omega_{1}, \omega_{2}}(\omega) \in \Omega$ for all $\omega \in \Omega$, and so $F(\Omega) \subset B(\Omega)$. In fact, $F(\Omega)$ and $F\left(\Omega_{\mathrm{wk}}\right)$ are algebraic ideals in $B(\Omega)$ and $B\left(\Omega_{\mathrm{wk}}\right)$ respectively. The norm-closures of these algebraic ideals, namely $K(\Omega)$ and $K\left(\Omega_{\mathrm{wk}}\right)$, are essential ideals in each of $B(\Omega)$ and $B\left(\Omega_{\mathrm{wk}}\right)$ —called the ideals of compact endomorphisms - and the multiplier algebras of $K(\Omega)$ and $K\left(\Omega_{\mathrm{wk}}\right)$ are, respectively, $B(\Omega)$ and $B\left(\Omega_{\mathrm{wk}}\right)[17]$.

When referring to rank-1 operators $x$ acting on a Hilbert space $H$, we will use the notation $x=\xi \otimes \eta$ for such an operator-the action on $\gamma \in H$ given by $\gamma \mapsto\langle\gamma, \eta\rangle \xi$-and we reserve the notation $\Theta_{\xi, \eta}$ for "rank-1" operators acting on a Hilbert module.

The term "homomorphism" will be used to mean a *-homomorphism between $\mathrm{C}^{*}$-algebras.

For any $C^{*}$-algebra $B$, we denote the injective envelope [13], [18, Chapter $15]$ of $B$ by $I(B)$ (and we consider $I(B)$ as a $C^{*}$-algebra rather than as an operator system).

The main result of the present section is the following. 
Theorem 3.1. There exist $C^{*}$-algebra embeddings such that

$$
K(\Omega) \subset A \subset B(\Omega) \subset B\left(\Omega_{\mathrm{wk}}\right)=I(K(\Omega)) .
$$

In particular, $I(K(\Omega))=I(A)=I(B(\Omega))=B\left(\Omega_{\mathrm{wk}}\right)$.

The proof of Theorem 3.1 and a description of the inclusions in (2) begin with the following set of results.

Lemma 3.2. For every $a \in A$ and $\omega \in \Omega$, the vector field $a \cdot \omega$ defined by $a \cdot \omega(s)=a(s) \omega(s)$ is an element of $\Omega$.

Proof. Let $a \in A$. Then $a^{*} a \in A_{+}$and since all fields in $A$ are weakly continuous, for every $\omega \in \Omega$ the map $s \mapsto\|a(s) \omega(s)\|=\left\langle a^{*} a \cdot \omega(s), \omega(s)\right\rangle^{1 / 2}$ is continuous.

Suppose $s_{0} \in \Delta$ and $\varepsilon>0$. Because $H_{s_{0}}=\left\{\mu\left(s_{0}\right): \mu \in \Omega\right\}$, there is a $\mu \in \Omega$ such that $a\left(s_{0}\right) \omega\left(s_{0}\right)=\mu\left(s_{0}\right)$. Since

$$
\|a \cdot \omega(s)-\mu(s)\|^{2}=\|a(s) \omega(s)\|^{2}+\|\mu(s)\|^{2}-2 \operatorname{Re}\langle a(s) \omega(s), \mu(s)\rangle
$$

is continuous on $\Delta$ and vanishes at $s_{0}$, there is an open set $U \subset \Delta$ containing $s_{0}$ such that $\|a \cdot \omega(s)-\mu(s)\|<\varepsilon$ for all $s \in U$. As $\Omega$ is closed under local uniform approximation, this proves that $a \cdot \omega \in \Omega$.

Proposition 3.3. The map $\varrho: A \rightarrow B(\Omega)$ given by $\varrho(a) \omega=a \cdot \omega$, for $a \in A$ and $\omega \in \Omega$ is an isometric homomorphism. Furthermore, $K(\Omega) \subset \varrho(A) \subset$ $B(\Omega)$ as $C^{*}$-algebras.

Proof. It is clear that $\varrho$ is a homomorphism, and so we only need to verify that it is one-to-one. To this end, assume that $\varrho(a)=0$. Thus, $a(s) \omega(s)=0$ for every $\omega \in \Omega$ and every $s \in \Delta$. Because $H_{s}=\{\omega(s): \omega \in \Omega\}$, this implies that $a(s)=0$ for all $s \in \Delta$, and so $a=0$.

To show $K(\Omega) \subset \varrho(A) \subset B(\Omega)$ as $\mathrm{C}^{*}$-algebras, consider $\Theta_{\omega_{1}, \omega_{2}}$ with $\omega_{1}, \omega_{2} \in \Omega$. The map $s \mapsto\left\|\Theta_{\omega_{1}(s), \omega_{2}(s)}\right\|$ is continuous because $\left\|\Theta_{\omega_{1}(s), \omega_{2}(s)}\right\|$ $=\left\|\omega_{1}(s)\right\|\left\|\omega_{2}(s)\right\|$. For any $\eta_{1}, \eta_{2} \in \Omega$, the map

$$
\left\langle\Theta_{\omega_{1}, \omega_{2}} \cdot \eta_{1}, \eta_{2}\right\rangle(s)=\left\langle\eta_{1}, \omega_{2}\right\rangle(s)\left\langle\omega_{1}, \eta_{2}\right\rangle(s)=\left\langle\eta_{1}(s), \omega_{2}(s)\right\rangle\left\langle\omega_{1}(s), \eta_{2}(s)\right\rangle
$$

is continuous. So $\Theta_{\omega_{1}, \omega_{2}}$ is also finite dimensional and weakly continuous, which shows that $\Theta_{\omega_{1}, \omega_{2}} \in A$ and $K(\Omega) \subset \varrho(A)$.

Lemma 3.4. With respect to the inclusion $\Omega \subset \Omega_{\mathrm{wk}}$, we have $\Omega^{\perp}=\{0\}$. 
Proof. Let $\nu \in \Omega_{\mathrm{wk}}$ be such that $\langle\nu, \omega\rangle=0$, for every $\omega \in \Omega$. That is, for every $\omega \in \Omega$ and for every $s \in \Delta,\langle\nu(s), \omega(s)\rangle=0$. If $\nu \neq 0$, there exists $s_{0} \in \Delta$ such that $\nu\left(s_{0}\right) \neq 0$. By axiom (II) in Definition 1.1, there exists $\omega \in \Omega$ such that $\omega\left(s_{0}\right)=\nu\left(s_{0}\right)$, in contradiction to $\left\langle\nu\left(s_{0}\right), \omega\left(s_{0}\right)\right\rangle=0$.

Lemma 3.5. If $t_{0} \in \Delta$ and $\xi \in H_{t_{0}}$, then there exists $\omega \in \Omega$ such that $\omega\left(t_{0}\right)=\xi$ and $\|\omega\|=\|\xi\|$.

Proof. The case $\xi=0$ is trivial. So assume that $\|\xi\|>0$. Let $\omega^{\prime} \in \Omega$ with $\omega^{\prime}\left(t_{0}\right)=\xi$. Fix a clopen neighbourhood $V$ of $t_{0}$ such that $V \subset\{t \in T$ : $\left.\left\|\omega^{\prime}(t)\right\| \geq\left\|\omega^{\prime}\left(t_{0}\right)\right\| / 2\right\}$. Let $h^{\prime}(\cdot)=\|\xi\| \cdot\left\|\omega^{\prime}(\cdot)\right\|^{-1} \in C(V)$; then $h^{\prime}$ extends to a continuous function $h \in C(\Delta)$ with $\left.h\right|_{\Delta \backslash V}=0$. It is now straightforward to show that $\omega=h \cdot \omega^{\prime} \in \Omega$ has the desired properties.

Proposition 3.6. There exists an isometric homomorphism $\vartheta: B(\Omega) \rightarrow$ $B\left(\Omega_{\mathrm{wk}}\right)$ such that for $a \in A, \nu \in \Omega_{\mathrm{wk}}$,

$$
(\vartheta(\varrho(a)) \nu)(s)=a(s) \nu(s), \quad s \in \Delta .
$$

Proof. Assume that $b \in B(\Omega)$ and $\omega \in \Omega, s \in \Delta$. By Lemma 3.5,

$$
\begin{aligned}
\|(b \omega)(s)\| & =\sup _{\xi \in H_{s},\|\xi\|=1}|\langle(b \omega)(s), \xi\rangle|=\sup _{\eta \in \Omega,\|\eta\|=1}|\langle(b \omega)(s), \eta(s)\rangle| \\
& =\sup _{\eta \in \Omega,\|\eta\|=1}|\langle b \omega, \eta\rangle(s)|=\sup _{\eta \in \Omega,\|\eta\|=1}\left|\left\langle\omega(s),\left(b^{*} \eta\right)(s)\right\rangle\right| \\
& \leq\|\omega(s)\| \sup _{\eta \in \Omega,\|\eta\|=1}\left\|b^{*} \eta\right\| \leq\|\omega(s)\|\left\|b^{*}\right\|=\|\omega(s)\|\|b\| .
\end{aligned}
$$

Therefore the function $\omega(s) \mapsto(b \omega)(s)$ is well defined and induces a bounded linear operator $b(s) \in B\left(H_{s}\right)$ such that $(b \omega)(s)=b(s) \omega(s)$, for $s \in \Delta$ and $\omega \in \Omega$, with $\sup _{s \in \Delta}\|b(s)\| \leq\|b\|$. Moreover,

$$
\begin{aligned}
\|b\| & =\sup _{\|\omega\|=1}\|b \cdot \omega\|=\sup _{\|\omega\|=1} \sup _{s}\|b \cdot \omega(s)\|=\sup _{\|\omega\|=1} \sup _{s}\|b(s) \omega(s)\| \\
& \leq \sup _{\|\omega\|=1} \sup _{s}\|b(s)\|\|\omega(s)\| \leq \sup _{s}\|b(s)\| \leq\|b\|,
\end{aligned}
$$

and so $\sup _{s \in \Delta}\|b(s)\|=\|b\|$. Suppose now that $\nu \in \Omega_{\mathrm{wk}}$ and $s \in \Delta$, and define a vector field $\vartheta b \nu$ by $(\vartheta b \nu)(s)=b(s) \nu(s)$. If $\eta \in \Omega$, then

$$
\langle(\vartheta b \nu)(s), \eta(s)\rangle=\left\langle\nu(s), b(s)^{*} \eta(s)\right\rangle=\left\langle\nu(s),\left(b^{*} \eta\right)(s)\right\rangle
$$

is continuous, which shows that $\vartheta b \nu$ is weakly continuous with respect to $\Omega$. Since $\vartheta b \nu$ is also uniformly bounded, we conclude that $\vartheta b \nu \in \Omega_{\mathrm{wk}}$. 
It is straightforward to show that the map $\nu \mapsto \vartheta b \nu$ is a bounded $C(\Delta)$ endomorphism of $\Omega_{\mathrm{wk}}$ and hence it gives rise to an element $\vartheta b \in B\left(\Omega_{\mathrm{wk}}\right)$. It is clear that $\vartheta$ is a homomorphism. If $\vartheta b=0$, then $b(s) \omega(s)=0$ for all $\omega \in \Omega, s \in \Delta$ and so $b(s)=0$ for all $s$; then $\|b\|=\sup _{s}\|b(s)\|=0$, and $b=0$. So $\vartheta$ is one-to-one, and thus isometric. Finally, it is clear that (3) holds by construction.

One consequence of the proof of Proposition 3.6 is that for every $b \in$ $B(\Omega)$ there exists an operator field $\{b(s)\}_{s \in \Delta}$ acting on the Hilbert bundle $\left\{H_{s}\right\}_{s \in \Delta}$ such that $(b \omega)(s)=b(s) \omega(s)$, for every $s \in \Delta$. This property, however, is not shared by all elements of $B\left(\Omega_{\mathrm{wk}}\right)$.

Lemma 3.7. If $z \in B\left(\Omega_{\mathrm{wk}}\right)$ and $\Theta_{\omega, \omega} z \Theta_{\mu, \mu}=0$ for all $\omega, \mu \in \Omega$, then $z=0$.

Proof. For any $\xi, \omega, \mu \in \Omega$ we have that

$$
0=\Theta_{\omega, \omega} z \Theta_{\mu, \mu} \xi=\langle\xi, \mu\rangle\langle z \mu, \omega\rangle \omega .
$$

Hence, we get that

$$
0=\langle\xi, \mu\rangle|\langle z \mu, \omega\rangle|^{2}=\langle\xi, \mu\rangle\left|\left\langle\mu, z^{*} \omega\right\rangle\right|^{2} .
$$

We are free to choose $\xi, \mu \in \Omega$. Fix $s$, and choose $\mu$ with $\mu(s)=z^{*} \omega(s)$; let $\xi=\mu$. Then, as $\mu \in \Omega$, we get $0=\langle\mu, \mu\rangle(s)=\langle\mu(s), \mu(s)\rangle$, so $z^{*} \omega(s)=$ $\mu(s)=0$. As $s \in \Delta$ is arbitrary, $z^{*} \omega=0$ for every $\omega \in \Omega$. For any $\nu \in \Omega_{\mathrm{wk}}$ and every $\omega \in \Omega,\langle z \nu, \omega\rangle=\left\langle\nu, z^{*} \omega\right\rangle=0$. By Lemma 3.4 we conclude that $z \nu=0$ for $\nu \in \Omega_{\mathrm{wk}}$ and hence $z=0$.

Proof of Theorem 3.1. We consider the embeddings $A \stackrel{\varrho}{\rightarrow} B(\Omega)$ and $B(\Omega) \stackrel{\vartheta}{\rightarrow}$ $B\left(\Omega_{\mathrm{wk}}\right)$ defined in Propositions 3.3 and 3.6. In this way, we get the inclusions in $(2)$.

Because $B\left(\Omega_{\mathrm{wk}}\right)$ is a type I $\mathrm{AW}^{*}$-algebra, it is injective [14, Proposition 5.2]. To show that $B\left(\Omega_{\mathrm{wk}}\right)$ is the injective envelope $I(K(\Omega))$ of $K(\Omega)$, we need to show that the embedding $\vartheta \circ \varrho$ of $K(\Omega)$ into $B\left(\Omega_{\mathrm{wk}}\right)$ is rigid [18, Theorem 15.8]: that is, we aim to prove that if $\phi: B\left(\Omega_{\mathrm{wk}}\right) \rightarrow B\left(\Omega_{\mathrm{wk}}\right)$ is a unital completely positive linear map for which $\left.\phi\right|_{K(\Omega)}=\operatorname{id}_{K(\Omega)}$, then $\phi=\mathrm{id}_{B\left(\Omega_{\mathrm{wk}}\right)}$.

Let $\phi: B\left(\Omega_{\mathrm{wk}}\right) \rightarrow B\left(\Omega_{\mathrm{wk}}\right)$ be such a ucp map with $\left.\phi\right|_{K(\Omega)}=\mathrm{id}_{K(\Omega)}$. Suppose that $z \in B\left(\Omega_{\mathrm{wk}}\right)$ and $\omega, \mu \in \Omega$. Then $\Theta_{\omega, \omega} z \Theta_{\mu, \mu}=\Theta_{\langle z \mu, \omega\rangle \omega, \mu} \in$ $K(\Omega)$. Because $K(\Omega)$ is in the multiplicative domain of $\phi$, we have that $\phi(a x b)=a \phi(x) b$ for all $x \in B\left(\Omega_{\mathrm{wk}}\right)$ and $a, b \in K(\Omega)$. This implies that

$$
\Theta_{\omega, \omega} \phi(z) \Theta_{\mu, \mu}=\phi\left(\Theta_{\omega, \omega} z \Theta_{\mu, \mu}\right)=\phi\left(\Theta_{\langle z \mu, \omega\rangle \omega, \mu}\right)=\Theta_{\langle z \mu, \omega\rangle \omega, \mu}=\Theta_{\omega, \omega} z \Theta_{\mu, \mu},
$$


and so $\Theta_{\omega, \omega}(z-\phi(z)) \Theta_{\mu, \mu}=0$. Since $\omega, \mu$ were arbitrary, Lemma 3.7 implies that $z-\phi(z)=0$ and so $\phi=\operatorname{id}_{B\left(\Omega_{\mathrm{wk}}\right)}$.

We have shown above that the inclusion $K(\Omega) \subset B\left(\Omega_{\mathrm{wk}}\right)$ is rigid. Moreover, $K(\Omega)$ is an essential ideal of $B(\Omega)$ and $K(\Omega) \subset A \subset B(\Omega)$. Hence, $I(K(\Omega))=I(A)=I(B(\Omega))=B\left(\Omega_{\mathrm{wk}}\right)$.

We conclude this section with a remark about the ideal $K\left(\Omega_{\mathrm{wk}}\right)$ of $B\left(\Omega_{\mathrm{wk}}\right)$. In type I $\mathrm{AW}^{*}$-algebras, the ideal generated by the abelian projections has a prominent role. As it happens, $K\left(\Omega_{\mathrm{wk}}\right)$ is precisely this ideal.

Proposition 3.8. The $C^{*}$-algebra $K\left(\Omega_{\mathrm{wk}}\right)$ coincides with the ideal $J \subset$ $B\left(\Omega_{\mathrm{wk}}\right)$ generated by the abelian projections of $B\left(\Omega_{\mathrm{wk}}\right)$. So $K\left(\Omega_{\mathrm{wk}}\right)$ is a liminal $C^{*}$-algebra with Hausdorff spectrum.

Proof. By [16, Lemma 13], a projection $e \in B\left(\Omega_{\mathrm{wk}}\right)$ is abelian if and only if there exists $\nu \in \Omega_{\mathrm{wk}}$ such that $|\nu|$ is a projection in $C(\Delta)$ and $e=\Theta_{\nu, \nu}$. Hence, $J \subset K\left(\Omega_{\mathrm{wk}}\right)$.

To show that $K\left(\Omega_{\mathrm{wk}}\right) \subset J$, assume $\nu \in \Omega_{\mathrm{wk}}$ is nonzero. Let $\varepsilon>0$. We will show that there is an $x_{\varepsilon} \in J$ such that $\left\|\Theta_{\nu, \nu}-x_{\varepsilon}\right\|<\varepsilon$. Let $V \subset \Delta$ be the (clopen) closure of $\left\{s \in \Delta:|\nu|(s)<\varepsilon^{1 / 2}\right\}, U=\Delta \backslash V$ (also clopen) and let $g=(1 /|\nu|) \chi_{U} \in C(\Delta)_{+}$. Then $g|\nu|=\chi_{U}$ and $\left\|\chi_{\Delta \backslash U}|\nu|\right\|<\varepsilon^{1 / 2}$. Let $\nu^{\prime}=g \cdot \nu$ so that $\left|\nu^{\prime}\right|=\chi_{U}$. Hence, $\Theta_{\nu^{\prime}, \nu^{\prime}} \in J$ and $\Theta_{\nu^{\prime}, \nu^{\prime}}=g^{2} \cdot \Theta_{\nu, \nu}$. Let $x_{\varepsilon}=|\nu|^{2} \cdot \Theta_{\nu^{\prime}, \nu^{\prime}} \in J$. Then

$$
x_{\varepsilon}=|\nu|^{2} \cdot \Theta_{\nu^{\prime}, \nu^{\prime}}=|\nu|^{2} g^{2} \Theta_{\nu, \nu}=\chi_{U} \Theta_{\nu, \nu},
$$

and $x_{\varepsilon}-\Theta_{\nu, \nu}=\chi_{\Delta \backslash U} \cdot \Theta_{\nu, \nu}$. Then

$$
\begin{aligned}
\left\|x_{\varepsilon}-\Theta_{\nu, \nu}\right\| & =\sup _{\eta \in\left(\Omega_{\mathrm{wk}}\right)_{1}}\left\|\chi_{\Delta \backslash U} \cdot \Theta_{\nu, \nu} \eta\right\|=\sup _{\eta \in\left(\Omega_{\mathrm{wk}}\right)_{1}}\left\|\chi_{\Delta \backslash U} \cdot\langle\eta, \nu\rangle \nu\right\| \\
& =\sup _{\eta \in\left(\Omega_{\mathrm{wk}}\right)_{1}} \max _{s \in \Delta \backslash U}|\langle\eta, \nu\rangle(s)|\|\nu(s)\| \\
& \leq \sup _{\eta \in\left(\Omega_{\mathrm{wk}}\right)_{1}} \max _{s \in \Delta \backslash U}|\eta|(s)|\nu|(s)\left|\|\nu(s)\| \leq \max _{s \in \Delta \backslash U}\right| \nu \mid(s)^{2}<\varepsilon .
\end{aligned}
$$

As $\varepsilon$ was arbitrary and $J$ is closed, we conclude that $\Theta_{\nu, \nu} \in J$. The polarisation identity then shows that $\Theta_{\nu_{1}, \nu_{2}} \in J$ for all $\nu_{1}, \nu_{2} \in \Omega_{\mathrm{wk}}$. Hence, $F\left(\Omega_{\mathrm{wk}}\right) \subset J$, and so $K\left(\Omega_{\mathrm{wk}}\right) \subset J$.

It remains to justify the last assertion in the statement. By the main result of [12], the ideal generated by the abelian projections in a type I $\mathrm{AW}^{*}$-algebra is liminal and has Hausdorff spectrum. Hence, this is true of $K\left(\Omega_{\mathrm{wk}}\right)$. 


\section{Multiplier and Local Multiplier Algebras}

In the previous section we established the inclusions $K(\Omega) \subset A \subset B(\Omega) \subset$ $B\left(\Omega_{\mathrm{wk}}\right)$, as $\mathrm{C}^{*}$-subalgebras, and we showed that $I(A)=B\left(\Omega_{\mathrm{wk}}\right)$. The present section refines these inclusions to incorporate multiplier algebras and local multiplier algebras.

Given a $\mathrm{C}^{*}$-algebra $C$, we denote by $M(C)$ and $M_{\mathrm{loc}}(C)$ its multiplier and local multiplier algebra [2] respectively.

The second order local multiplier algebra of $C$ is $M_{\text {loc }}\left(M_{\text {loc }}(C)\right)$, the local multiplier algebra of $M_{\text {loc }}(C)$. By [11, Corollary 4.3], the local multiplier algebras (of all orders) of $C$ are $\mathrm{C}^{*}$-subalgebras of the injective envelope $I(C)$ of $C$. In particular, $C \subset M_{\text {loc }}(C) \subset M_{\text {loc }}\left(M_{\text {loc }}(C)\right) \subset I(C)$ as $\mathrm{C}^{*}$ subalgebras.

By a well known theorem of Kasparov [2, Theorem 1.2.33], [17, Theorem 2.4], $M(K(\Omega))=B(\Omega)$. We remark that all the subalgebras we consider are essential in $B\left(\Omega_{\mathrm{wk}}\right)$ (i.e. the annihilator is zero), and so whenever we write $M(C)$ for one of these subalgebras $C \subset B\left(\Omega_{\mathrm{wk}}\right)$, we mean the concrete realization $[20]$

$$
M(C)=\left\{x \in B\left(\Omega_{\mathrm{wk}}\right): x C+C x \subset C\right\} .
$$

The following theorem is the main result of this section.

Theorem 4.1. With the notations from the previous sections, we have the equality $M_{\mathrm{loc}}(A)=M_{\mathrm{loc}}\left(K(\Omega)\right.$ ) and the following inclusions (as $C^{*}$ subalgebras):

$$
\begin{aligned}
M(A) & \subset M(K(\Omega))=B(\Omega) \\
& \subset M_{\mathrm{loc}}(K(\Omega)) \subset M_{\mathrm{loc}}\left(M_{\mathrm{loc}}(K(\Omega))\right)=B\left(\Omega_{\mathrm{wk}}\right) .
\end{aligned}
$$

In particular, $M_{\mathrm{loc}}\left(M_{\mathrm{loc}}(A)\right)=I(A)$.

Ara and Mathieu have presented examples of Stonean spaces $\Delta$ and trivial Hilbert bundles $\Omega$ where the inclusion $M_{\text {loc }}(K(\Omega)) \subset M_{\text {loc }}\left(M_{\text {loc }}(K(\Omega))\right)$ in (4) is proper [3, Theorem 6.13]. As a consequence of Theorem 4.1 and the fact that $B\left(\Omega_{\mathrm{wk}}\right)=I(K(\Omega))$, we see that this gap cannot occur for higher local multiplier algebras, i.e. for all $k \geq 2, M_{\mathrm{loc}}^{k+1}(K(\Omega))=M_{\mathrm{loc}}^{k}(K(\Omega))$ where $M_{\mathrm{loc}}^{k+1}(K(\Omega))=M_{\mathrm{loc}}\left(M_{\mathrm{loc}}^{k}(K(\Omega))\right)$ for $k \geq 1$.

The proof of Theorem 4.1 is achieved through a number of lemmas. 
Lemma 4.2. The set

$$
F_{+}=\left\{\sum_{j=1}^{n} \Theta_{\omega_{j}, \omega_{j}}: n \in \mathbb{N}, \omega_{j} \in \Omega\right\}
$$

is dense in the positive cone of $K(\Omega)$.

Proof. Assume that $h \in K(\Omega)_{+}$and let $\varepsilon>0$ be arbitrary. For each $s_{0} \in \Delta$ consider the positive compact operator $h\left(s_{0}\right) \in K\left(H_{s_{0}}\right)$. Then there are vectors $\xi_{1}, \ldots, \xi_{n_{s_{0}}} \in H_{s_{0}}$ such that

$$
\left\|h\left(s_{0}\right)-\sum_{j=1}^{n_{s_{0}}} \xi_{j} \otimes \xi_{j}\right\|<\varepsilon .
$$

Using (II) in Definition 1.1, choose $\omega_{1}, \ldots, \omega_{n_{s_{0}}} \in \Omega$ such that $\omega_{j}\left(s_{0}\right)=\xi_{j}$, $1 \leq j \leq n_{s_{0}}$, and let $\kappa_{s_{0}}=\sum_{j=1}^{n_{s_{0}}} \Theta_{\omega_{j}, \omega_{j}}$. By continuity of the operator fields in $A$, there is an open set $U_{s_{0}} \subset \Delta$ containing $s_{0}$ such that $\left\|h(s)-\kappa_{s_{0}}(s)\right\|<\varepsilon$ for all $s \in U_{s_{0}}$.

This procedure leads to an open cover $\left\{U_{s}\right\}_{s \in \Delta}$ of $\Delta$, from which (by compactness) there exists a finite subcover $\left\{U_{1}, \ldots, U_{m}\right\}$ and corresponding fields $\kappa_{i}=\sum_{j=1}^{n_{i}} \Theta_{\omega_{j}^{[i]}, \omega_{j}^{[i]}}$. Let $\left\{\psi_{1}, \ldots, \psi_{m}\right\} \subset C(\Delta)$ be a partition of unity subordinate to $\left\{U_{1}, \ldots, U_{m}\right\}$ and note that $\psi_{i} \cdot \Theta_{\omega_{j}^{[i]}, \omega_{j}^{[i]}}=\Theta_{\psi_{i}^{1 / 2} \cdot \omega_{j}^{[i]}, \psi_{i}^{1 / 2} \cdot \omega_{j}^{[i]}}$ for all $j$ and $i$. Hence, the field $\kappa=\sum_{i=1}^{m} \psi_{i} \cdot \kappa_{i}$ is in $F_{+}$, and for each $s \in \Delta$,

$$
\|h(s)-\kappa(s)\|=\left\|\sum_{i=1}^{m} \psi_{i} \cdot\left(h-\kappa_{i}\right)(s)\right\| \leq \sum_{i=1}^{m} \psi_{i}(s)\left\|\left(h-\kappa_{i}\right)(s)\right\|<\varepsilon .
$$

Hence, $h$ is in the norm-closure of $F_{+}$.

Lemma 4.3. Let $\left\{U_{i}\right\}_{i \in \Lambda}$ be a family of pairwise disjoint clopen subsets of $\Delta$ whose union $U$ is dense in $\Delta$, and let $c_{i}=\chi_{U_{i}} \in C(\Delta)$, for each $i \in \Lambda$. Suppose that $\left\{\omega_{i}\right\}_{i \in \Lambda}$ is any bounded family in $\Omega$ and let $\tilde{\omega}=\sum_{i \in \Lambda} c_{i} \omega_{i} \in$ $\Omega_{\mathrm{wk}}$, in the sense of Remark 2.5. If $f \in C(\Delta)$ is such that $f(s)=0$ for $s \in \Delta \backslash U$, then $f \cdot \tilde{\omega} \in \Omega$.

Proof. Fix $s_{0} \in \Delta$ and let $\varepsilon>0$. If $s_{0} \in \Delta \backslash U$, then by the continuity of $f$ and the fact that $f\left(s_{0}\right)=0$ there exists an open subset $U_{s_{0}} \subset \Delta$ containing $s_{0}$ such that $|f(s)|<\varepsilon\|\tilde{\omega}\|^{-1}$ for all $s \in U_{s_{0}}$. Hence, the vector field $f \cdot \tilde{\omega}$ is within $\varepsilon$ of the zero vector field $0 \in \Omega$ on the open set $U_{s_{0}}$.

On the other hand, if $s_{0} \in U$, then there exists $j \in \Lambda$ such that $s_{0} \in U_{j}$. By construction, $c_{j} \cdot \tilde{\omega}=c_{j} \cdot \omega_{j}$ and so $\tilde{\omega}(s)=\omega_{j}(s)$ for all $s \in U_{j}$. Because 
$\left\|(f \cdot \tilde{\omega})(s)-\left(f \cdot \omega_{j}\right)(s)\right\|=0$ for all $s \in U_{j}$, the vector field $f \cdot \tilde{\omega}$ is within $\varepsilon$ of the vector field $f \cdot \omega_{j} \in \Omega$ on the open set $U_{j}$. Thus, by the local uniform approximation property (axiom (IV) in Definition 1.1), $f \cdot \tilde{\omega} \in \Omega$.

The fact that $\Omega^{\perp}=\{0\}$ in $\Omega_{\mathrm{wk}}$ (Lemma 3.4) suggests that $\Omega$ is somehow dense in $\Omega_{\mathrm{wk}}$. The next proposition makes this relation more explicit.

Proposition 4.4. If $\nu \in \Omega_{\mathrm{wk}}$ and $\varepsilon>0$, then there exist a family $\left\{c_{i}\right\}_{i \in \Lambda}$ of pairwise orthogonal projections in $C(\Delta)$ with supremum 1 and a bounded family $\left\{\omega_{i}\right\}_{i \in \Lambda} \subset \Omega$ such that $\left\|\nu-\sum_{i \in \Lambda} c_{i} \cdot \omega_{i}\right\|<\varepsilon$.

Proof. By Lemma 2.2, the function $s \mapsto\|\nu(s)\|$ is lower semicontinuous; hence, there exists a meagre set $M_{\nu}$ such that the function $s \mapsto\|\nu(s)\|$ is continuous in the relative topology of $\Delta \backslash M_{\nu}$. Observe that $\overline{\left(\Delta \backslash M_{\nu}\right)}=\Delta$.

Fix $s_{0} \in \Delta \backslash M_{\nu}$ and let $\omega \in \Omega$ be such that $\omega\left(s_{0}\right)=\nu\left(s_{0}\right)$. Since

$$
\|\nu(s)-\omega(s)\|^{2}=\|\nu(s)\|^{2}+\|\omega(s)\|^{2}-2 \operatorname{Re}\langle\nu, \omega\rangle(s),
$$

the continuity in the relative topology of $\Delta \backslash M_{\nu}$ guarantees the existence of an open subset $U_{s_{0}}$ of $\Delta$ containing $s_{0}$ such that $\|\nu(s)-\omega(s)\|<\varepsilon / 2$ for all $s \in\left(\Delta \backslash M_{\nu}\right) \cap U_{s_{0}}$. Hence, again by continuity we get that $\|\nu-\omega\|(s)<\varepsilon$ for all $s \in \bar{U}_{s_{0}}$. The set $\bar{U}_{s_{0}}$ is a clopen subset of $\Delta$ and $\Delta^{\prime}=\Delta \backslash \bar{U}_{s_{0}}$ is also a Stonean space. Further, $M_{\nu} \cap \Delta^{\prime}=M_{\nu} \cap\left(\Delta \backslash \bar{U}_{s_{0}}\right)$ is a meagre set such that the function $s \mapsto\|\nu(s)\|$, for $s \in \Delta^{\prime} \backslash\left(M_{\nu} \cap \Delta^{\prime}\right)$, is continuous in the relative topology.

An application of Zorn's Lemma yields a maximal family $\left\{\left(\chi_{U_{i}}, \omega_{i}\right)\right\}_{i \in \Lambda}$ such that $U_{i} \cap U_{j}=\emptyset$ for $i \neq j$ and such that $\left\|\chi_{U_{i}}\left(\nu-\omega_{i}\right)\right\|<\varepsilon$. Maximality ensures that $\overline{\left(\cup_{i \in I} U_{i}\right)}=\Delta$, for otherwise we can enlarge this family by the previous procedure in the Stonean space $\Delta \backslash \overline{\left(\cup_{i \in \Lambda} U_{i}\right)}$. If we let $c_{i}=\chi_{U_{i}}$ for $i \in \Lambda$ then it is clear by Lemma 2.2 that $\left\|\nu-\sum_{i \in \Lambda} c_{i} \cdot \omega_{i}\right\|<\varepsilon$ as for every $j \in \Lambda$ we have that $\left\|c_{j}\left(\nu-\sum_{i \in \Lambda} c_{i} \cdot \omega_{i}\right)\right\|=\left\|c_{j}\left(\nu-\omega_{j}\right)\right\|<\varepsilon$ and $\bigvee_{i \in \Lambda} c_{i}=1$.

The next result is the key step in the proof of Theorem 4.1.

Proposition 4.5. For every abelian projection $e \in B\left(\Omega_{\mathrm{wk}}\right)$ and $\varepsilon>0$ there is an essential ideal $I \subset K(\Omega)$ and $x \in M(I)$ such that $\|e-x\|<\varepsilon$.

Proof. Assume that $e \in B\left(\Omega_{\mathrm{wk}}\right)$ is an abelian projection and let $\varepsilon>0$. Thus, by [16, Lemma 13], $e=\Theta_{\nu, \nu}$ for some $\nu \in \Omega_{\mathrm{wk}}$ for which $\langle\nu, \nu\rangle$ is a projection of $C(\Delta)$. By Proposition 4.4, there is a family $\left\{c_{i}\right\}_{i \in \Lambda}$ of pairwise orthogonal projections in $C(\Delta)$ with supremum 1 and a bounded family 
$\left\{\omega_{j}\right\}_{j \in \Lambda} \subset \Omega$ such that $\|\nu-\tilde{\omega}\|<\varepsilon /(2\|\nu\|)$, where $\tilde{\omega}=\sum_{j \in \Lambda} c_{j} \cdot \omega_{j} \in \Omega_{\mathrm{wk}}$. Each $c_{j}$ is the characteristic function of a clopen set $U_{j}$ and the union $U$ of these sets $U_{j}$ is dense in $\Delta$.

Let $I=\{a \in K(\Omega): a(s)=0, \forall s \in \Delta \backslash U\}$, which is an essential ideal of $K(\Omega)$. Define $F^{I} \subset F_{+} \subset K(\Omega)_{+}$to be the set

$$
F^{I}=\left\{\sum_{i=1}^{n} \Theta_{\mu_{i}, \mu_{i}}: n \in \mathbb{N}, \mu_{i} \in \Omega,\left.\mu_{i}\right|_{\Delta \backslash U}=0, i=1, \ldots, n\right\} .
$$

Suppose that $\eta \in \Omega$ satisfies $\|\eta(s)\|=0$ for all $s \in \Delta \backslash U$, and consider $\Theta_{\eta, \eta} \in F^{I}$. Observe that $\Theta_{\tilde{\omega}, \tilde{\omega}} \Theta_{\eta, \eta}=\Theta_{\langle\eta, \tilde{\omega}\rangle \cdot \tilde{\omega}, \eta}$, which is an element of $I$ because $\langle\eta, \tilde{\omega}\rangle(s)=\langle\eta(s), \tilde{\omega}(s)\rangle=0$ for all $s \in \Delta \backslash U$ and $\langle\eta, \tilde{\omega}\rangle \cdot \tilde{\omega} \in \Omega$ by Lemma 4.3. Hence, $\Theta_{\tilde{\omega}, \tilde{\omega}}$ maps the set $F^{I}$ back into $I$. Because $F^{I}$ is dense in $I_{+}$, as we shall show below, $\Theta_{\tilde{\omega}, \tilde{\omega}} I \subset I$ and a similar computation shows that $I \Theta_{\tilde{\omega}, \tilde{\omega}} \subset I$. Furthermore, writing $x=\Theta_{\tilde{\omega}, \tilde{\omega}}$,

$$
\|e-x\|=\left\|\Theta_{\nu, \nu}-\Theta_{\tilde{\omega}, \tilde{\omega}}\right\| \leq(\|\nu\|+\|\tilde{\omega}\|)\|\nu-\tilde{\omega}\|<\varepsilon .
$$

It remains to show that $F^{I}$ is dense in $I_{+}$. To this end, assume $\varepsilon^{\prime}>0$ and $\kappa \in I_{+}$. Thus, $\kappa(s)=0$ for all $s \in \Delta \backslash U$. Furthermore, by Lemma 4.2, there exists $h \in F_{+}$such that $\|\kappa-h\|<\varepsilon^{\prime}$. Let $\tilde{h}=\chi_{\Delta \backslash U} \cdot h$ and note that, as $\kappa \in I$, it is also true that $\|\kappa-\tilde{h}\|<\varepsilon^{\prime}$. Now if $h$ has the form $\sum_{j=1}^{n} \Theta_{\mu_{j}, \mu_{j}}$ for some $\mu_{j} \in \Omega$, then $\tilde{h}=\sum_{j=1}^{n} \Theta_{\chi_{\Delta \backslash U} \mu_{j}, \chi_{\Delta \backslash U} \mu_{j}} \in F^{I}$.

Proof of Theorem 4.1. Because $K(\Omega)$ is an ideal of $A$, we have $M(A) \subset$ $M(K(\Omega))$. Moreover, as $K(\Omega)$ is an essential ideal of $A$ we conclude that $M_{\mathrm{loc}}(A)=M_{\mathrm{loc}}(K(\Omega))[2$, Proposition 2.3.6]. On the other hand, the inclusions

$$
B(\Omega)=M(K(\Omega)) \subset M_{\mathrm{loc}}(K(\Omega)) \subset M_{\mathrm{loc}}\left(M_{\mathrm{loc}}(K(\Omega))\right) \subset B\left(\Omega_{\mathrm{wk}}\right)
$$

hold by [11, Theorem 4.6].

Therefore, we are left to show that $M_{\mathrm{loc}}\left(M_{\mathrm{loc}}(K(\Omega))\right)=B\left(\Omega_{\mathrm{wk}}\right)$. By [11, Corollary 4.3], an element $z \in I(K(\Omega))=B\left(\Omega_{\mathrm{wk}}\right)$ belongs to $M_{\mathrm{loc}}(K(\Omega))$ if and only if for every $\varepsilon>0$ there is an essential ideal $I \subset K(\Omega)$ and a multiplier $x \in M(I)$ such that $\|z-x\|<\varepsilon$. By Proposition 3.8, $K\left(\Omega_{\mathrm{wk}}\right)$ is the (essential) ideal of $B\left(\Omega_{\mathrm{wk}}\right)$ generated by the abelian projections of $B\left(\Omega_{\mathrm{wk}}\right)$; thus, by Proposition $4.5, K\left(\Omega_{\mathrm{wk}}\right) \subset M_{\mathrm{loc}}(K(\Omega))$. Hence, $K\left(\Omega_{\mathrm{wk}}\right)$ is an essential ideal of $M_{\mathrm{loc}}(K(\Omega))$ and so $M\left(K\left(\Omega_{\mathrm{wk}}\right)\right) \subset M_{\mathrm{loc}}\left(M_{\mathrm{loc}}(K(\Omega))\right)$. However, $B\left(\Omega_{\mathrm{wk}}\right)=M\left(K\left(\Omega_{\mathrm{wk}}\right)\right)$ by Kasparov's Theorem [17, Theorem 2.4] (or by a theorem of Pedersen [20]); hence,

$$
B\left(\Omega_{\mathrm{wk}}\right)=M\left(K\left(\Omega_{\mathrm{wk}}\right)\right) \subset M_{\mathrm{loc}}\left(M_{\mathrm{loc}}(K(\Omega))\right) \subset B\left(\Omega_{\mathrm{wk}}\right),
$$


which yields $M_{\mathrm{loc}}\left(M_{\mathrm{loc}}(K(\Omega))\right)=B\left(\Omega_{\mathrm{wk}}\right)$.

Somerset has shown that every separable postliminal (that is, type I) $\mathrm{C}^{*}$ algebra $A$ has the property that $M_{\mathrm{loc}}\left(M_{\mathrm{loc}}(A)\right)=I(A)$ [22, Theorem 2.8]. Theorem 4.1 demonstrates that the same behavior occurs with (certain) nonseparable type I $\mathrm{C}^{*}$-algebras. Somerset's methods are different from ours in at least two ways: he employs the Baire $*$-envelope of a $\mathrm{C}^{*}$-algebra where we use the injective envelope and he uses properties of Polish spaces - spaces that arise from the separability of the algebras under study. It is reasonable to conjecture that $M_{\mathrm{loc}}\left(M_{\mathrm{loc}}(A)\right)=I(A)$ for all $\mathrm{C}^{*}$-algebras $A$ that possess a postliminal essential ideal. To prove such a statement, it would be enough to prove it for any continuous trace $\mathrm{C}^{*}$-algebra $A$.

\section{Direct Sum Decompositions}

A Kaplansky-Hilbert module $E$ over $C(\Delta)$ is said to be homogeneous [16] if there is a subset $\left\{\nu_{j}\right\}_{j \in \Lambda} \subset E$ - called an orthonormal basis - such that $\left\langle\nu_{i}, \nu_{j}\right\rangle=0$ for all $j \neq i,\left|\nu_{j}\right|=1$ for all $j$, and $\left\{\nu_{j}\right\}_{j \in \Lambda}^{\perp}=\{0\}$, where for any $\nu \in E,|\nu|$ is the continuous real-valued function $|\nu|=\langle\nu, \nu\rangle^{1 / 2} \in C(\Delta)$.

Kaplansky introduced the notion of homogeneous $\mathrm{AW}^{*}$-module with the aim of reducing the study of abstract $\mathrm{AW}^{*}$-modules to the slightly more concrete setting in which the modules have an orthonormal basis. This is justified by the following result:

Theorem 5.1 ([16]). Let $E$ be a Kaplansky-Hilbert module over $C(\Delta)$. Then there exist orthogonal projections $\left\{c_{i}\right\}_{i \in I} \subset C(\Delta)$ with supremum 1 such that $c_{i} E$ is a homogenous $A W^{*}$-module over $c_{i} C(\Delta)$.

Note that in the situation of Theorem 5.1, for each $i$ there exists a clopen set $\Delta_{i} \subset \Delta$ with $c_{i}=\chi_{\Delta_{i}}$. The sets $\left\{\Delta_{i}\right\}$ are pairwise disjoint, and $\cup_{i} \Delta_{i}$ is dense in $\Delta$.

In this section we consider the effect of a direct sum decomposition in the structures that have been studied in the previous sections, namely the Fell algebra $A$ of the weakly continuous Hilbert bundle $\left(\Delta,\left\{H_{s}\right\}_{s \in \Delta}, \Omega, \Omega_{\mathrm{wk}}\right)$, and its local multiplier algebra $M_{\mathrm{loc}}(A)$. We show that a decomposition of $\Omega_{\mathrm{wk}}$ into a direct sum $\oplus_{i} c_{i} \Omega_{\mathrm{wk}}$ given by a partition of the identity $\left\{c_{i}\right\}$ in $C(\Delta)$ leads one to consider two corresponding direct sum $\mathrm{C}^{*}$-algebras: $\oplus_{i} A_{i}$ and $\oplus_{i} M_{\text {loc }}\left(A_{i}\right)$, where $A_{i}$ is a subalgebra of $A$ for all $i$. We prove that $A$ need not be isomorphic to $\oplus_{i} A_{i}$, yet $M_{\text {loc }}(A) \cong \oplus_{i} M_{\text {loc }}\left(A_{i}\right)$. The latter result is especially interesting if one recalls that $M_{\mathrm{loc}}(A)$ is generally not an $\mathrm{AW}^{*}$-algebra [3, Theorem 6.13]. 
Theorem 5.2. Let $\left(\Delta,\left\{H_{s}\right\}_{s \in \Delta}, \Omega\right)$ be a continuous Hilbert bundle over the Stonean space $\Delta$. Assume that $\left\{\Delta_{i}\right\}_{i \in I}$ is a family of pairwise-disjoint clopen subsets of $\Delta$ whose union is dense in $\Delta$, and for each $i \in I$ let $c_{i}=\chi_{\Delta_{i}} \in C(\Delta)$ and $\Omega_{i}=\left\{\omega_{\mid \Delta_{i}}: \omega \in \Omega\right\}$. Then:

i. $\left(\Delta_{i},\left\{H_{s}\right\}_{s \in \Delta_{i}}, \Omega_{i}\right)$ is a continuous Hilbert bundle;

ii. $\left(\Omega_{i}\right)_{\mathrm{wk}} \cong c_{i} \cdot \Omega_{\mathrm{wk}}$ as $C^{*}$-modules;

iii. $\Omega_{\mathrm{wk}} \cong \bigoplus_{i}\left(\Omega_{i}\right)_{\mathrm{wk}}$ as $C^{*}$-modules;

iv. $B\left(\left(\Omega_{i}\right)_{\mathrm{wk}}\right) \cong c_{i} \cdot B\left(\Omega_{\mathrm{wk}}\right)$ as $C^{*}$-algebras;

v. $B\left(\Omega_{\mathrm{wk}}\right) \cong \bigoplus_{i} B\left(\left(\Omega_{i}\right)_{\mathrm{wk}}\right)$ as $C^{*}$-algebras.

In $\mathbf{i i}$ and $\mathbf{i i i}$, the isomorphism is considered together with the identification $C\left(\Delta_{i}\right) \simeq c_{i} C(\Delta)$.

Proof. Being clopen in $\Delta$, each $\Delta_{i}$ is itself a Stonean space, and it is easy to see that $C\left(\Delta_{i}\right) \cong c_{i} C(\Delta)$

$\boldsymbol{i}$. For axiom (I) in Definition 1.1, we aim to show that $\Omega_{i}$ is a $C\left(\Delta_{i}\right)$ module. Let $\omega \in \Omega$ and consider $\omega_{i}=\left.\omega\right|_{\Delta_{i}}$. Choose any $f_{i} \in C\left(\Delta_{i}\right)$. As $\Delta_{i}$ is clopen, $f_{i}$ can be extended to $F_{i} \in C(\Delta)$ such that $f_{i}=\left.F_{i}\right|_{\Delta_{i}}$, and $\left.F_{i}\right|_{\Delta \backslash \Delta_{i}}=0$. The action $f_{i} \cdot \omega_{i}=\left.\left(F_{i} \cdot \omega\right)\right|_{\Delta_{i}}$ gives $\Omega_{i}$ the structure of a $C\left(\Delta_{i}\right)$ module. Axioms (II) and (III) of Definition 1.1 are trivially satisfied.

For axiom (IV), let $\xi: \Delta_{i} \rightarrow \bigsqcup_{s \in \Delta_{i}} H_{s}$ be a vector field such that for every $s_{0} \in \Delta_{i}$ and $\varepsilon>0$ there is an open set $U_{i} \subset \Delta_{i}$ containing $s_{0}$ and a $\omega_{i} \in \Omega_{i}$ with $\left\|\omega_{i}(s)-\xi(s)\right\|<\varepsilon$ for all $s \in U_{i}$. Let $\Xi: \Delta_{i} \rightarrow \bigsqcup_{s \in \Delta} H_{s}$ be the vector field that coincides with $\xi$ on $\Delta_{i}$ and is identically zero off $\Delta_{i}$. By the definition of $\Omega_{i}$, there is $\omega \in \Omega$ such that $\omega_{i}=\left.\omega\right|_{\Delta_{i}}$. The set $U_{i}$ is also open in $\Delta$, and $\|\omega(s)-\Xi(s)\|<\varepsilon$ for all $s \in U_{i}$. If $s_{0} \notin \Delta_{i}$ choose any open set $V_{i}$ containing $s_{0}$ such that $V_{i} \cap U_{i}=\emptyset$ and let $\omega \in \Omega$ be arbitrary; then $0=\left\|\chi_{\Delta_{i}}(s) \omega(s)-\Xi(s)\right\|<\varepsilon$ for all $s \in V_{i}$. Since $\chi_{\Delta_{i}} \cdot \omega \in \Omega$ and since $\Omega$ is closed under local uniform approximation, $\Xi \in \Omega$, whence $\xi \in \Omega_{i}$.

ii. Let $T_{i}: c_{i} \Omega_{\mathrm{wk}} \rightarrow\left(\Omega_{i}\right)_{\mathrm{wk}}$ be given by $T_{i}\left(c_{i} \nu\right)=\left.\nu\right|_{\Delta_{i}}$. It is clear that $T_{i}$ is well defined, linear, bounded, and has trivial kernel; to show that it is onto, note that if $\nu_{i} \in\left(\Omega_{i}\right)_{\mathrm{wk}}$, then-since $\Delta_{i}$ is clopen - the vector field $\nu: \Delta \rightarrow \bigsqcup_{s \in \Delta} H_{s}$ defined by $\nu(s)=0$, for $s \notin \Delta_{i}$, and $\nu(s)=\nu_{i}(s)$, for $s \in \Delta_{i}$, has the property that $\langle\omega, \nu\rangle \in C(\Delta)$, for all $\omega \in \Omega$; so $\nu \in \Omega_{\mathrm{wk}}$ and $\nu_{i}=T_{i}\left(c_{i} \nu\right)$. It is also easy to check that $T_{i}$ preserves inner products.

iii. Let $T: \Omega_{\mathrm{wk}} \rightarrow \bigoplus_{i}\left(\Omega_{i}\right)_{\mathrm{wk}}$, given by $T \nu=\left(T_{i}\left(c_{i} \nu\right)\right)_{i \in I}$. The previous paragraph and Lemma 2.1 show that $T$ is an isometry; we show now that $T$ 
is onto. Suppose that $\nu^{\prime}=\left(\nu_{i}\right)_{i \in I} \in \bigoplus_{i}\left(\Omega_{i}\right)_{\mathrm{wk}}$. For each $i \in I$ let $\tilde{\nu}_{i}$ denote the vector field on $\Delta$ that coincides with $\nu_{i}$ on $\Delta_{i}$ and vanishes elsewhere. Then $\tilde{\nu}_{i} \in \Omega_{\mathrm{wk}}$ and $T_{i}\left(c_{i} \tilde{\nu}_{i}\right)=\nu_{i}$. Hence, if $\nu=\sum_{i} c_{i} \tilde{\nu}_{i}$ as in Remark 2.5, we have $\nu \in \Omega_{\mathrm{wk}}$ and $T \nu=\nu^{\prime}$. Thus, $\Omega_{\mathrm{wk}}$ and $\bigoplus_{i}\left(\Omega_{i}\right)_{\mathrm{wk}}$ are isomorphic Banach spaces. Similar arguments show that $\bigoplus_{i}\left(\Omega_{i}\right)_{\mathrm{wk}}$ is a $C(\Delta)$-module and that $T$ is module isomorphism. Hence, $\Omega_{\mathrm{wk}} \cong \bigoplus_{i}\left(\Omega_{i}\right)_{\mathrm{wk}}$ as $\mathrm{C}^{*}$-modules. $i v$. Let $\rho_{i}: c_{i} B\left(\Omega_{\mathrm{wk}}\right) \rightarrow B\left(\left(\Omega_{i}\right)_{\mathrm{wk}}\right)$ be given by $\rho_{i}\left(c_{i} b\right) T_{i}\left(c_{i} \nu\right)=\left.(b \nu)\right|_{\Delta_{i}}$. This map is well-defined because if $c_{i} b_{1}=c_{i} b_{2}$ then for any $\nu \in \Omega_{\mathrm{wk}}$ we have $\left.\left(b_{1} \nu\right)\right|_{\Delta_{i}}=\left.\left(c_{i} b_{1} \nu\right)\right|_{\Delta_{i}}=\left.\left(c_{i} b_{2} \nu\right)\right|_{\Delta_{i}}=\left.\left(b_{2} \nu\right)\right|_{\Delta_{i}}$. A similar computation shows that $\rho_{i}$ is one-to-one, and linearity is clear. To see that $\rho_{i}$ is onto, let $b_{i} \in B\left(\left(\Omega_{i}\right)_{\mathrm{wk}}\right)$. Consider the injection ${ }^{\sim}:\left(\Omega_{i}\right)_{\mathrm{wk}} \rightarrow \Omega_{\mathrm{wk}}$ where $\tilde{\nu_{i}} \in \Omega_{\mathrm{wk}}$ is the vector field that agrees with $\nu_{i}$ on $\Delta_{i}$ and is 0 elsewhere. Let $b \in B\left(\Omega_{\mathrm{wk}}\right)$ be the operator given by $b \nu=\widehat{b_{i}\left(\left.\nu\right|_{\Delta_{i}}\right)}$. Then $\rho_{i}\left(c_{i} b\right)\left(T_{i} c_{i} \nu\right)=\left.(b \nu)\right|_{\Delta_{i}}=$ $\left.\widehat{b_{i}\left(\left.\nu\right|_{\Delta_{i}}\right)}\right|_{\Delta_{i}}=b_{i}\left(\left.\nu\right|_{\Delta_{i}}\right)=b_{i}\left(T_{i} c_{i} \nu\right)$, so $\rho_{i}\left(c_{i} b\right)=b_{i}$.

$\boldsymbol{v}$. Let $\rho: B\left(\Omega_{\mathrm{wk}}\right) \rightarrow \bigoplus_{i} B\left(\left(\Omega_{i}\right)_{\mathrm{wk}}\right)$ be the map $\rho(b)=\left(\rho_{i}\left(c_{i} b\right)\right)_{i \in I}$. It is clear that $\rho$ is a homomorphism. If $\rho(b)=0$ for some $b \in B\left(\Omega_{\mathrm{wk}}\right)$, then - as each $\rho_{i}$ is one-to-one $-c_{i} b=0$ for all $i$; this implies that $b^{*} b=$ $b^{*}\left(\sup _{i}\left(c_{i} \cdot I\right)\right) b=\sup _{i}\left(b^{*} c_{i} b\right)=0$ by [14, Corollary 4.10], so $b=0$ and $\rho$ is one-to-one. To show that $\rho$ is onto, let $\left(b_{i}\right)_{i} \in \bigoplus_{i} B\left(\left(\Omega_{i}\right)_{\mathrm{wk}}\right)$; as each $\rho_{i}$ is onto, there exist operators $b^{i} \in B\left(\Omega_{\mathrm{wk}}\right)$ with $\rho_{i}\left(c_{i} b^{i}\right)=b_{i}$. Define $b \in B\left(\Omega_{\mathrm{wk}}\right.$ ) by $b \nu=\sum_{i} c_{i} b^{i} \nu$ (in the sense of Remark 2.5; that is, $c_{i} b \nu=$ $\left.c_{i} b^{i} \nu\right)$. Then $\left.\rho_{i}\left(c_{i} b\right) \nu\right|_{\Delta_{i}}=\left.\left(c_{i} b \nu\right)\right|_{\Delta_{i}}=\left.\left(c_{i} b^{i} \nu\right)\right|_{\Delta_{i}}=\left.\rho_{i}\left(c_{i} b^{i}\right) \nu\right|_{\Delta_{i}}=\left.b_{i} \nu\right|_{\Delta_{i}}$. So $\rho(b)=\left(b_{i}\right)_{i}$.

Proposition 5.3. Assume the notation, hypotheses, and conclusions of Theorem 5.2. Then there exists an example where the canonical embedding $\Omega \hookrightarrow \bigoplus_{i} \Omega_{i}$ (via the isometry $T$ from the proof of $\boldsymbol{i i i}$ in Theorem 5.2) is not onto. In particular, $\Omega$ is properly contained in $\Omega_{\mathrm{wk}}$.

Proof. Take $\Delta$ and the family of clopen subsets $\left\{\Delta_{i}\right\}_{i \in I}$ in Theorem 5.2 to be such that $\bigcup_{i \in I} \Delta_{i} \neq \Delta$. Thus, $I$ is an infinite set. Let $H$ be a Hilbert space with orthonormal basis $\left\{e_{i}\right\}_{i \in I}$ and consider the trivial Hilbert bundle $\Omega=C(\Delta, H)$ of all continuous functions $\omega: \Delta \rightarrow H$. As in Theorem 5.2, let $\Omega_{i}=C\left(\Delta_{i}, H\right)$.

For each $i \in I$, set $\omega_{i} \in \Omega$ with $\omega_{i}(s)=e_{i}$ for all $s$ and consider $\left(\omega_{i}\right)_{i \in I} \in \bigoplus_{i} \Omega_{i}$. Under the isomorphism of Theorem 5.2, this element $\left(\omega_{i}\right)_{i \in I}$ is identified with $\omega=\sum_{i \in I} \chi_{\Delta_{i}} \cdot \tilde{\omega}_{i} \in \Omega_{\mathrm{wk}}$ (in the sense of Remark $2.5)$, where $\tilde{\omega}_{i}$ is any element of $\Omega$ that agrees with $\omega_{i}$ on $\Delta_{i}$ and vanishes off $\Delta_{i}$. Under this identification, $\omega \notin \Omega$; that is, the function $s \mapsto\|\omega(s)\|$ fails to be continuous on $\Delta$. We argue this by contradiction. 
Assume that $s \mapsto\|\omega(s)\|$ is continuous on $\Delta$. Because $\|\omega(s)\|=1$ for all $s \in \cup_{i \in I} \Delta_{i}$, continuity implies that $\|\omega(s)\|=1$ for $s \in \Delta$. Choose $s_{0} \in \Delta \backslash\left(\cup_{i \in I} \Delta_{i}\right)$ and let $\left(s_{\alpha}\right)_{\alpha \in \Lambda} \subset \cup_{i \in I} \Delta_{i}$ be a net such that $s_{\alpha} \rightarrow s_{0}$. Let $\eta \in \Omega$ be the constant field $\eta(s)=\omega\left(s_{0}\right)$, for all $s \in \Delta$. Since $\omega \in \Omega_{\mathrm{wk}}$, we have

$$
\lim _{\alpha}\left\langle\omega\left(s_{\alpha}\right), \eta\left(s_{\alpha}\right)\right\rangle=\left\langle\omega\left(s_{0}\right), \eta\left(s_{0}\right)\right\rangle=\left\langle\omega\left(s_{0}\right), \omega\left(s_{0}\right)\right\rangle=1 .
$$

For each $\alpha \in \Lambda$ let $i(\alpha) \in I$ be such that $s_{\alpha} \in \Delta_{i(\alpha)}$. Thus, for every $\alpha \in \Lambda$, $I_{\alpha}=\{i(\beta): \beta \in I, \beta \geq \alpha\}$ is an infinite set (for otherwise $s_{0} \in \Delta_{i}$ for some $i \in I)$. Therefore,

$$
\lim _{\alpha}\left\langle\omega\left(s_{\alpha}\right), \eta\left(s_{\alpha}\right)\right\rangle=\lim _{\alpha}\left\langle e_{i(\alpha)}, \omega\left(s_{0}\right)\right\rangle=0 .
$$

As (5) and (6) cannot be true simultaneously, we obtain a contradiction. Hence, $\omega \notin \Omega$.

Our second reduction theorem below notes some consequences of Theorem 5.2 when applied to the injective envelope and local multiplier algebras of the Fell algebra $A$ associated to a continuous Hilbert bundle.

Theorem 5.4. Let $\left(\Delta,\left\{H_{t}\right\}_{t \in \Delta}, \Omega\right)$ be a continuous Hilbert bundle over the Stonean space $\Delta$ and let $A=\left(\Delta,\left\{K\left(H_{t}\right\}, \Gamma\right)\right.$ denote the associated continuous trace $C^{*}$-algebra of Fell. Assume that $\left\{\Delta_{i}\right\}_{i \in I}$ is a family of pairwisedisjoint clopen subsets of $\Delta$ whose union is dense in $\Delta$, and for each $i \in I$ let $c_{i}=\chi_{\Delta_{i}} \in C(\Delta)$ and $\Omega_{i}=\left\{\omega_{\mid \Delta_{i}}: \omega \in \Omega\right\}$. Then:

i. if $A_{i}$ denotes the Fell algebra of $\left(\Delta_{i},\left\{H_{s}\right\}_{s \in \Delta_{i}}, \Omega_{i}\right)$, then $A_{i} \cong c_{i} \cdot A$;

ii. $I\left(A_{i}\right)=B\left(\left(\Omega_{i}\right)_{\mathrm{wk}}\right)$;

iii. $I(A) \cong \bigoplus_{i \in I} I\left(A_{i}\right)$;

iv. $M_{\mathrm{loc}}(A) \cong \bigoplus_{i \in I} M_{\mathrm{loc}}\left(A_{i}\right)$.

Proof. Let $A_{i}=\left(\Delta_{i},\left\{K\left(H_{s}\right)\right\}_{s \in \Delta}, \Gamma_{i}\right)$ denote the Fell C*-algebra associated to the Hilbert bundle $\left(\Delta_{i},\left\{H_{s}\right\}_{s \in \Delta_{i}}, \Omega_{i}\right)$. That is, $\Gamma_{i}$ consists of all weakly continuous almost finite-dimensional operator fields $a_{i}: \Delta_{i} \rightarrow \bigsqcup_{s \in \Delta_{i}} K\left(H_{s}\right)$ such that $s \mapsto\left\|a_{i}(s)\right\|$ is continuous. We have that $B\left(\left(\Omega_{i}\right)_{\mathrm{wk}}\right)$ is a type I $\mathrm{AW}^{*}$-algebra with centre $C\left(\Delta_{i}\right)$.

$\boldsymbol{i}$. For each $a_{i} \in \Gamma_{i}$ there is an $a \in \Gamma$ such that $a_{i}=\left.a\right|_{\Delta_{i}}$. To verify this, let $a: \Delta_{i} \rightarrow \bigsqcup_{s \in \Delta} K\left(H_{s}\right)$ be the operator field defined by $a(s)=a_{i}(s)$, for $s \in \Delta_{i}$, and $a(s)=0$, for $s \notin \Delta_{i}$. Since $\Delta_{i}$ is a clopen set, the maps 
$s \rightarrow\|a(s)\|$ and $s \mapsto\left\langle a(s) \omega_{1}(s), \omega_{2}(s)\right\rangle$ are continuous for every $\omega_{1}, \omega_{2} \in \Omega$. The operator field $a$ is also locally finite-dimensional, again because $\Delta_{i}$ is clopen and $a_{i}$ has the property on $\Delta_{i}$. Hence, $a \in \Gamma$. Next, let $\pi_{i}: A_{i} \rightarrow c_{i} A$ be defined by $\pi_{i}\left(a_{i}\right)=c_{i} a$, where $a \in A$ is any operator field that restricts to $a_{i}$ on $\Delta_{i}$. This map is clearly well-defined, and a homomorphism.

ii. By Theorem 3.1, $B\left(\left(\Omega_{i}\right)_{\mathrm{wk}}\right)=I\left(A_{i}\right)=I\left(c_{i} A\right)$.

iii. By $\left[14\right.$, Lemma 6.2], $I\left(c_{i} A\right)=c_{i} I(A)$. Hence, $I\left(A_{i}\right)=B\left(\left(\Omega_{i}\right)_{\mathrm{wk}}\right)$ and Theorem 5.2 immediately yields $I(A) \cong \bigoplus_{i \in I} I\left(A_{i}\right)$.

$\boldsymbol{i v}$. We take each $M_{\mathrm{loc}}\left(A_{i}\right)$ to be a $\mathrm{C}^{*}$-subalgebra of $B\left(\left(\Omega_{i}\right)_{\mathrm{wk}}\right)$. First we remark that the isomorphism $\rho$ from Theorem 5.2 sends $A$ into $\bigoplus_{i} A_{i}$. To see why, recall that $a \nu(s)=a(s) \nu(s)$, for all $a \in A, \nu \in \Omega_{\mathrm{wk}}$, and $s \in \Delta$ (Proposition 3.6). Since, for a given $i \in I$, the action of $\rho_{i}(a)$ on $\nu_{i} \in\left(\Omega_{i}\right)_{\mathrm{wk}}$ is defined by $\left.\nu_{i} \mapsto(a \nu)\right|_{\Delta_{i}}$, where $\nu \in \Omega_{\mathrm{wk}}$ is any vector with $\left.\nu\right|_{\Delta_{i}}=\nu_{i}$, it is easy to verify that $\rho_{i}(a)$ is a weakly continuous almost finite-dimensional operator field on $\Delta_{i}$.

To show that $\rho\left(M_{\text {loc }}(A)\right) \subset \bigoplus_{i} M_{\text {loc }}\left(A_{i}\right)$, let $x \in M_{\text {loc }}(A) \subset I(A)$ and suppose that $\varepsilon>0$. Thus, there is an essential ideal $J \subset A$ and a multiplier $x \in M(J)$ such that $\|x-y\|<\varepsilon$. Further, there exists an open dense subset $U \subset \Delta$ such that

$$
J=\{a \in A: a(s)=0, s \in \Delta \backslash U\} .
$$

For $i \in I$, let $U_{i}=\Delta_{i} \cap U$, which is an open dense set in $\Delta_{i}$. Therefore,

$$
J_{i}=\left\{a_{i} \in A_{i}: a(s)=0, s \in \Delta_{i} \backslash U_{i}\right\}
$$

is an essential ideal in $A_{i}$. We aim to show that $\rho_{i}(y) \in M\left(J_{i}\right)$. To this end, select $a_{i} \in J_{i}$. As $A_{i} \cong c_{i} \cdot A$, there is an $a \in A$ such that $a_{i}(s)=a(s)$ for all $s \in \Delta_{i}$. Moreover, $a \in A$ can be chosen so that $a(s)=0$ for all $s \in \Delta \backslash \Delta_{i}$.

Because $a_{i} \in J_{i}$, we conclude that $a(s)=0$ for all $s \in \Delta \backslash U$; that is, $a \in J$. Therefore, ya $\in J$, which implies that $y a(s)=0$ for all $s \in \Delta \backslash U$. In particular, $y a(s)=0$ for all $s \in \Delta_{i} \backslash U_{i}$. The element $\rho_{i}(y) a_{i} \in B\left(\left(\Omega_{i}\right)_{\mathrm{wk}}\right)$ is in fact an operator field since $\rho_{i}(y) a_{i}=\rho_{i}(y) \rho_{i}\left(c_{i} a\right)=\rho_{i}\left(c_{i}(y a)\right) \in A_{i}$. Then, for all $s \in \Delta_{i} \backslash U_{i}$ and $\nu \in \Omega_{\mathrm{wk}}$,

$$
\begin{aligned}
{\left[\rho_{i}(y) a_{i}\right](s)\left(T_{i} c_{i} \nu\right)(s) } & =\rho_{i}(y) a_{i}\left(T_{i} c_{i} \nu\right)(s)=\rho_{i}\left(c_{i} y a\right)\left(T_{i} c_{i} \nu\right)(s) \\
& =\left.(y a) \nu\right|_{\Delta_{i}}(s)=\left.(y a)(s) \nu\right|_{\Delta_{i}}(s)=0 .
\end{aligned}
$$

With $\nu$ being arbitrary, we conclude that $\rho_{i}(y) a_{i}(s)=0$, that is $\rho_{i}(y) a_{i} \in J_{i}$, and so $\rho_{i}(y)$ is a left multiplier of $J_{i}$. By a similar $\operatorname{argument,} \rho_{i}(y)$ is a right multiplier of $J_{i}$, and so $\rho_{i}(y) \in M\left(J_{i}\right)$. Thus, $\rho(y) \in \bigoplus_{i} M_{\mathrm{loc}}\left(A_{i}\right)$ and 
$\|\rho(x)-\rho(y)\|=\|x-y\|<\varepsilon$. As $\varepsilon>0$ was chosen arbitrarily, this proves that $\rho(x) \in \bigoplus_{i} M_{\text {loc }}\left(A_{i}\right)$.

Conversely, let us show that $\bigoplus_{i} M_{\text {loc }}\left(A_{i}\right) \subset \rho\left(M_{\text {loc }}(A)\right)$. Let $\left(x_{i}\right)_{i} \in$ $\bigoplus_{i} M_{\text {loc }}\left(A_{i}\right)$; thus for each $i \in I$, there exist an essential ideal $J_{i} \subset A_{i}$ and $y_{i} \in M\left(J_{i}\right)$ such that $\left\|x_{i}-y_{i}\right\|<\varepsilon$ for all $i \in I$. For each $i \in I$, there exists an open dense subset $U_{i} \subset \Delta_{i}$ such that $J_{i}$ is given as in (8). Define $U=\bigcup_{i \in I} U_{i}$, which is an open dense subset of $\Delta$ and let $J$ be the essential ideal of $A$ defined as in (7) (for our present choice of $U$ ). Let $y \in B\left(\Omega_{\mathrm{wk}}\right)$ be such that $\rho(y)=\left(y_{i}\right)_{i}$.

For each $\omega \in \Omega$, we have that $y \omega \in \Omega_{\mathrm{wk}}$.

Claim 1. If $\omega \in \Omega$ is such that $\omega(s)=0$ for all $s \in \Delta \backslash U$, then $y \omega \in \Omega$ and $y \omega(s)=0$ for $s \in \Delta \backslash U$.

Assuming Claim 1, consider the set $F_{+}=\operatorname{span}\left\{\Theta_{\omega, \omega}: \omega \in \Omega, \omega(s)=\right.$ 0 for $s \in \Delta \backslash U\}$, which by Lemma 4.2 is dense in $K_{+}$, where $K$ is the essential ideal of $K(\Omega)$ defined by $K=K(\Omega) \cap J$. By the Claim, $y \Theta_{\omega, \omega}=$ $\Theta_{y \omega, \omega} \in K$ for all $\omega \in \Omega$. Therefore, $y$ is a left multiplier of $K$. Similarly, $y$ is a right multiplier of $K$, which yields $y \in M(K)$. Hence, $\left(x_{i}\right)_{i \in I}$ is within $\varepsilon$ of a multiplier-namely, $\rho(y)$ - of an essential ideal of $\rho(K(\Omega)$ ). Thus, by the Frank-Paulsen description of local multiplier algebras [11], $\left(x_{i}\right)_{i \in I} \in \rho\left(M_{\mathrm{loc}}(K(\Omega))\right)$. By Theorem 4.1, $M_{\mathrm{loc}}(A)=M_{\mathrm{loc}}(K(\Omega))$, so $\left(x_{i}\right)_{i \in I} \in \rho\left(M_{\mathrm{loc}}(A)\right)$.

We are now left with proving Claim 1. Assume that $\omega \in \Omega$ with $\omega(s)=0$ for all $s \in \Delta \backslash U$. Let $i \in I$ and let $\omega_{i}=\left.\omega\right|_{\Delta_{i}} \in \Omega_{i}$. Note that for every $\eta_{i} \in \Omega_{i}, \Theta_{\omega_{i}, \eta_{i}} \in J_{i}$, and hence $\Theta_{y_{i} \omega_{i}, \eta_{i}}=y_{i} \Theta_{\omega_{i}, \eta_{i}} \in J_{i}$. Also, $y_{i} \omega_{i} \in \Omega_{i}$. Indeed, suppose that $s_{0} \in \Delta_{i}$ and let $\eta_{i} \in \Omega_{i}$ such that $\left\|\eta_{i}\left(s_{0}\right)\right\|=1$. Choose a clopen subset $V_{i} \subset \Delta_{i}$ of $s_{0}$ for which $\left\|\eta_{i}(s)\right\| \geq 1 / 2$ for all $s \in V_{i}$ and define $f(s)=\chi_{V_{i}}(s)\left\|\eta_{i}(s)\right\|^{-2}$. Thus, $f \in C\left(\Delta_{i}\right)$ and so $f \cdot \eta_{i} \in \Omega_{i}$. Then, since $\Theta_{y_{i} \omega_{i}, \eta_{i}} \in J_{i} \subset A_{i}$, we have $\Theta_{y_{i} \omega_{i}, \eta_{i}}\left(f \cdot \eta_{i}\right) \in \Omega_{i}$. So $\chi_{V_{i}} \cdot y_{i} \omega_{i}=$ $\Theta_{y_{i} \omega_{i}, \eta_{i}}\left(f \cdot \eta_{i}\right) \in \Omega_{i}$. Thus, $y_{i} \omega_{i}$ is a local uniform limit of vectors fields in $\Omega_{i}$ and hence, $y_{i} \omega_{i} \in \Omega_{i}$. Moreover, since $\Theta_{y_{i} \omega_{i}, \eta_{i}} \in J_{i}$ for any $\eta_{i} \in \Omega_{i}$, we have $y_{i} \omega_{i}(s)=0$ for $s \in \Delta_{i} \backslash U_{i}$.

Since $(y \omega)(s)=\left(y_{i} \omega_{i}\right)(s)$ for $s \in \Delta_{i}$, the lower semicontinuous function $s \mapsto\|(y \omega)(s)\|$ is continuous on $\bigcup_{i} \Delta_{i}$ and vanishes on $\left(\bigcup_{i} \Delta_{i}\right) \backslash U$.

Claim 2. There exists $C>0$ such that $\|y \omega(s)\| \leq C\|\omega(s)\|, s \in \Delta_{i}$, $i \in I$.

We will use Claim 2 to show that the function $s \mapsto\|(y \omega)(s)\|$ is continuous on $\Delta$. Let $s \in \Delta \backslash\left(\bigcup_{i} \Delta_{i}\right)$ and let $\left(s_{\alpha}\right)_{\alpha} \subset \bigcup_{i} \Delta_{i}$ be a net such that $s_{\alpha} \rightarrow s$ in $\Delta$. This implies that $\lim _{\alpha}\left\|\omega\left(s_{\alpha}\right)\right\|=0$. By lower semicontinuity 
of the function $s \mapsto\|(y \omega)(s)\|$,

$$
0 \leq\|y \omega(s)\| \leq \lim _{\alpha}\left\|y \omega\left(s_{\alpha}\right)\right\| \leq C \lim _{\alpha}\left\|\omega\left(s_{\alpha}\right)\right\|=0,
$$

and it follows that $s \mapsto\|(y \omega)(s)\|$ is continuous on $\Delta$ and vanishes in $\Delta \backslash U$. This establishes Claim 1.

We finish the proof by proving Claim 2. Fix $s \in \Delta_{i}$, and let $C=$ $\sup _{i}\left\|y_{i}\right\|$. We already know that $y_{i} \omega_{i} \in \Omega_{i}$, and so

$$
\begin{aligned}
\|y \omega(s)\| & =\left\|y_{i} \omega_{i}(s)\right\|=\left\|y_{i} \omega_{i}\right\|(s) \leq\left\|y_{i}\right\|\left\|\omega_{i}\right\|(s) \\
& \leq C\left\|\omega_{i}\right\|(s)=C\left\|\omega_{i}(s)\right\|=C\|\omega(s)\| . \square
\end{aligned}
$$

Local multiplier algebras behave well under direct sums: $M_{\mathrm{loc}}\left(\oplus_{i} A_{i}\right) \cong$ $\oplus_{i} M_{\mathrm{loc}}\left(A_{i}\right)$ [2, Proposition 2.3.6]. However, the isomorphism of local multiplier algebras in Theorem 5.4 cannot be established via that generic result:

Proposition 5.5. Assume the notation, hypotheses, and conclusions of Theorem 5.4. Although $\rho$ sends $A$ into $\bigoplus_{i} A_{i}$, it need not be true that $A \cong \bigoplus_{i} A_{i}$.

Proof. If $\Delta$ and $\Omega$ are as in Proposition 5.3, then $\rho\left(\Theta_{\omega, \omega}\right)=\left(\Theta_{\omega_{i}, \omega_{i}}\right)_{i \in I} \in$ $\oplus_{i \in I} A_{i}$, but $\rho\left(\Theta_{\omega, \omega}\right) \notin \rho(A)$.

\section{References}

[1] P. Ara and M. Mathieu. A simple local multiplier algebra. Math. Proc. Cambridge Philos. Soc., 126(3):555-564, 1999.

[2] P. Ara and M. Mathieu. Local multipliers of $C^{*}$-algebras. Springer Monographs in Mathematics. Springer-Verlag London Ltd., London, 2003.

[3] P. Ara and M. Mathieu. Maximal $C^{*}$-algebras of quotients and injective envelopes of $C^{*}$-algebras. Houston J. Math., 34(3):827-872, 2008.

[4] M. Argerami, D. Farenick, and P. Massey. The gap between local multiplier algebras of $C^{*}$-algebras. Q. J. Math., 60(3):273-281, 2009.

[5] S. K. Berberian. Baer *-rings. Springer-Verlag, New York, 1972. Die Grundlehren der mathematischen Wissenschaften, Band 195.

[6] G. F. Birkenmeier, J. K. Park, and S. T. Rizvi. Hulls of semiprime rings with applications to $C^{*}$-algebras. J. Algebra, 322(2):327-352, 2009. 
[7] D. P. Blecher and V. I. Paulsen. Multipliers of operator spaces, and the injective envelope. Pacific J. Math., 200(1):1-17, 2001.

[8] J. Dixmier and A. Douady. Champs continus d'espaces hilbertiens et de $C^{*}$-algèbres. Bull. Soc. Math. France, 91:227-284, 1963.

[9] R. Dumitru, C. Peligrad, and B. Vişinescu. Automorphisms inner in the local multiplier algebra and Connes spectrum. In Operator theory 20, volume 6 of Theta Ser. Adv. Math., pages 75-80. Theta, Bucharest, 2006 .

[10] J. M. G. Fell. The structure of algebras of operator fields. Acta Math., 106:233-280, 1961.

[11] M. Frank and V. I. Paulsen. Injective envelopes of $C^{*}$-algebras as operator modules. Pacific J. Math., 212(1):57-69, 2003.

[12] H. Halpern. The maximal GCR ideal in an AW*-algebra. Proc. Amer. Math. Soc., 17:906-914, 1966.

[13] M. Hamana. Injective envelopes of $C^{*}$-algebras. J. Math. Soc. Japan, 31(1):181-197, 1979.

[14] M. Hamana. Regular embeddings of $C^{*}$-algebras in monotone complete $C^{*}$-algebras. J. Math. Soc. Japan, 33(1):159-183, 1981.

[15] M. Hamana. Tensor products for monotone complete $C^{*}$-algebras. I, II. Japan. J. Math. (N.S.), 8(2):259-283, 285-295, 1982.

[16] I. Kaplansky. Modules over operator algebras. Amer. J. Math., 75:839858, 1953.

[17] E. C. Lance. Hilbert $C^{*}$-modules, volume 210 of London Mathematical Society Lecture Note Series. Cambridge University Press, Cambridge, 1995. A toolkit for operator algebraists.

[18] V. Paulsen. Completely bounded maps and operator algebras, volume 78 of Cambridge Studies in Advanced Mathematics. Cambridge University Press, Cambridge, 2002.

[19] G. K. Pedersen. Approximating derivations on ideals of $C^{*}$-algebras. Invent. Math., 45(3):299-305, 1978.

[20] G. K. Pedersen. Multipliers of AW*-algebras. Math. Z., 187(1):23-24, 1984. 
[21] D. W. B. Somerset. The local multiplier algebra of a $C^{*}$-algebra. Quart. J. Math. Oxford Ser. (2), 47(185):123-132, 1996.

[22] D. W. B. Somerset. The local multiplier algebra of a $C^{*}$-algebra. II. J. Funct. Anal., 171(2):308-330, 2000.

[23] H. Takemoto. On the weakly continuous constant field of Hilbert space and its application to the reduction theory of von Neumann algebra. Tôhoku Math. J. (2), 28(3):479-496, 1976.

[24] M. Takesaki. Theory of operator algebras. I, volume 124 of Encyclopaedia of Mathematical Sciences. Springer-Verlag, Berlin, 2002. Reprint of the first (1979) edition, Operator Algebras and Non-commutative Geometry, 5 . 